\title{
TWO-YEAR CLINICAL PERFORMANCE AND MARGINAL INTEGRITY EVALUATION OF HIGH C-FACTOR OCCLUSAL CAVITIES RESTORED WITH PREHEATED BULK-FILL, SONICFILL AND INCREMENTAL FILL COMPOSITE RESIN
}

\author{
Reham Mohamad Attia*
}

\begin{abstract}
Aim of the Study: To study the clinical performance and marginal integrity of high C-factor Occlusal Cavities restored with preheated Bulk-Fill, SonicFill and incremental fill composite resin.

Materials and Methods: Thirty patients who required at least a triplet of class I restorations were included in the study. The patients were divided into 3 groups; Group I: Restored with preheated bulk-fill composite, Group II: Restored with SonicFill composite and Group III: Restored with Nano-hybrid incremental composite. The studied composites were evaluated clinically for the following clinical parameters: marginal integrity and adaptation, marginal discoloration, color match and surface roughness, wear, retention of restoration, presence of secondary caries and post-operative sensitivity. Restorations were evaluated at baseline, six, 12, 18 and 24 months using modified US Public Health Service Ryge criteria and recorded as Alpha, Bravo, or Charlie. Where, Alpha corresponds to excellent, Bravo to clinically acceptable, and Charlie to clinically unacceptable results.
\end{abstract}

Results: Regarding the marginal integrity, there was no statistically significant difference amongst the three tested materials except after 18 months, $(\mathrm{P}$-value $=0.026)$. There was statistically significant difference amongst the three tested groups at 18- and 24-months evaluation where $(\mathrm{P}$-value $=0.031)$ and $(\mathrm{P}$-value $=0.034)$, respectively. No statistical significance was observed between the 3 groups regarding wear, color match, surface roughness, retention of restoration, presence of secondary caries and post-operative sensitivity.

Conclusion: All tested materials showed comparable satisfactory clinical results with the exception of marginal integrity.

KEYWORDS: Clinical Performance, Marginal Integrity, Preheated Bulk-Fill, SonicFill, Incremental fill composite resin

\footnotetext{
* Assistant Professor, Conservative Dentistry Department, Faculty of Dentistry and Oral Medicine, Zagazig University.
} 


\section{INTRODUCTION}

Recently, there is a significant advancement in the physical and optical properties of the composite resins, though some of the residual restrictions such as polymerization shrinkage, and the resultant polymerization stress ${ }^{[1]}$.

Bulk-Fill composite resins are novel materials used for restoration. The Bulk-Fill composite resins can be inserted in the cavity in one increment up to $4 \mathrm{~mm}-5 \mathrm{~mm}$ in thickness without affecting the degree of conversion or physical properties of the material ${ }^{[2]}$. The superior translucency, filler concentrations and the use of photoactivation systems have a major impact on the enhancement of polymerization ${ }^{[3]}$. In addition to this, the bulk polymerization of Bulk-Fill composite resin is likely because of difference in its chemical components that decreased polymerization stress ${ }^{[4]}$. These alterations have permitted superior conversion at deep cavities ${ }^{[5]}$.

Pre-heating of uncured composites resin is one of the methods that are used as a technique to increase the composite resin material handling characteristics during restoration ${ }^{[6]}$. Increasing the temperature of composites preceding to curing usually reduces their viscosity, leading to improve marginal integrity. In addition decrease microleakage by reason of enhanced wetting of cavity walls ${ }^{[7]}$. Preheating enhances the radical and monomer mobility leading to alteration in many mechanical and physical properties as high diametral, tensile, flexural strength and surface roughness ${ }^{[8]}$.

Regarding the effect of preheating on pulp, Karacan and Ozyurt, concluded that the preheating of composite resin led to rise of the temperature intra-pulpally, nevertheless this increase is not the serious factor that causes damage and injury to the pulp ${ }^{[9]}$. Though, opportunity of pulpal damage produced by increase in temperature should be considered when using light-cured preheated BulkFill composite materials in very deep cavities ${ }^{[10]}$.

SonicFill (Kerr) is one of the Bulk-illF composites, which depends on the insertion of composite in the cavity preparation by sonic activation using a SonicFill handpiece ${ }^{[11]}$. Sonic activation leads to a noteworthy decline in the composite material viscosity and thus enhancing the adaptation of the composite material to the cavity wall and margins ${ }^{[12]}$. Sonic-Fill is a unique type of Bulk-Fill composite resin which can be photopolymerized with a depth up to $5 \mathrm{~mm}$ in only $20 \mathrm{sec}$ using a high-intensity light-curing unit ${ }^{[13]}$. The material is contoured and sculpted previous to photopolymerization ${ }^{[14]}$.

$\mathrm{C}$-factor is measured as the ratio of bonded surface area to unbonded area. Increasing $\mathrm{C}$-factor means that the ratio of free surfaces decreases. So, the ability of material to flow and subsequent stress release is reduced ${ }^{[15]}$.

The most important factors affecting the restorations' clinical success is the sealing of the margins and its adaptation as well as the type of adhesive system used in bonding procedure ${ }^{[16]}$. Other factors include: the degree of shrinkage stresses, finishing and polishing of the restoration ${ }^{[17]}$. Presence of gaps at the resin-tooth interface are signs of future composite restorations' failure since the establishment of micro gaps could lead to grow microleakage over time and cause bacterial and water penetration, which may lead to discoloration, postoperative sensitivity, and eventually restoration failure when the gaps are in the range of $0.5-1.0 \mu \mathrm{m}$ or larger ${ }^{[18]}$.

Penetration of marginal gaps by bacteria is probable. On the other hand, smaller gaps counteract bacterial penetration but may allow the diffusion of toxins and other bacterial products that can activate the immune system, provide chemotactic stimuli and induce cytokine production ${ }^{[19]}$.

Thus, the aim of this study was to evaluate the clinical performance of preheated Bulk-Fill composite, SonicFill and conventional incremental fill composite in addition to assessment of gap formation at the restorative material-tooth interface using replica and scanning electron microscopy examination in high $\mathrm{C}$-factor occlusal cavity. 


\section{MATERIAL AND METHODS}

Ethical clearance and patient consent were obtained from the institutional ethical review committee prior to the beginning of the study . Thirty patients [18 males \& 12 females] of the age group (25-45) who required at least a triplet of class I restorations were included in the study. The inclusion criteria were: Patients having vital teeth with a normal appearance and morphology and sound occlusal and interproximal contacts with adjacent teeth, good oral hygiene, good periodontal condition, absence of damaging habits (e.g. bruxism, nail biting, tooth clenching and mouth breathing), and the existence of at least three similar sized (as possible) primary carious lesions in molar and premolar teeth. Teeth with secondary caries or in need of replacement of existing restorations were not included in the study. Exclusion criteria were: patients with known allergic or adverse reaction to the tested materials used in the study, poor oral hygiene, severe or chronic periodontitis, nonvital, fractured, or cracked teeth, defective restorations adjacent to or opposite the tooth restored in the study, rampant caries, atypical extrinsic staining of teeth, any uncontrolled systemic disease, pregnant or lactating women and any patient incapable to be present at recall follow up visits.

They were diagnosed clinically and radiographically for class I caries, and the teeth were then randomly divided into 3 groups as follows:

Group I (no= 30 posterior teeth) class I cavity preparations were restored with preheated Bulk-Fill composite (Tetric N- Ceram Bulk-Fill composite)

Group II (no=30 posterior teeth) class I cavity preparations were restored with Sonicfill composite (SonicFill)

Group III (no $=30$ posterior teeth) the cavities restored with incremental fill composite (Tetric ${ }^{\circledR}$ $\mathrm{N}$-Ceram Nano-hybrid incremental composite).
The Bulk-Fill composites either preheated or SonicFill were considered the experimental group, whereas the incremental fill composite was the control group.

All the materials and its composition are listed in (Table 1). The distribution of materials and tooth locations were randomized by tossing a coin. The distribution of teeth restored in relation to different tested groups was shown in (Table 2).

All patients understood the background of the current study and the clinical steps were completed by the same operator. To permit an intra-individual comparison each patient received at least three restorations. Clinical procedure of each group was done as follows:

Group I: Carious lesion was removed under continuous water cooling. The cavosurface angle of the prepared cavity was entirely in enamel without any beveling. The operative field was carefully isolated with cotton rolls and suction device.

A three-step etch-and-rinse adhesive OptiBond FL (Kerr Co, Orange, CA, USA), was used according to the manufacturer's instructions. $37.5 \%$ phosphoric acid (Kerr Co, Orange, CA, USA) was applied to the enamel and dentin for a period of 30 $\mathrm{s}$ and $15 \mathrm{~s}$, respectively. Subsequently the cavities were then rinsed with an air/water spray for $15 \mathrm{~s}$. A layer of primer was applied to the dentin for 30 $\mathrm{sec}$, afterward gentle air-drying for $5 \mathrm{sec}$. Then, the bond was applied on the enamel and dentin and light cured with a LED curing unit (Bluephase, Ivoclar Vivadent, Liechtenstein) at $650 \mathrm{~mW} / \mathrm{cm} 2$ for about $30 \mathrm{sec}$.

Composite (Tetric N- Ceram Bulk-Fill composite) was preheated to temperature $54^{\circ} \mathrm{C}$ using Calset warmer device (Calset TM AdDent, Inc. Danbury, CT USA)). The composite was then applied in one bulk and cured for $20 \mathrm{sec}$ (Bluephase N Ivoclar Vivadent ). Carbon paper was used to detect pre- 
mature contacts and occlusal adjustments. The restorations were finished under water-cooling with finishing burs and finally polishing was performed using discs (Opti Disc, Kerr.) and rubber points (HiLusterPLUS Polishing System, Kerr).

Group II: Cavity preparation and bonding procedure was done exactly as it was in group I. The SonicFill composite resin (Kerr Co, Orange, CA, USA), was inserted into the cavity preparation in one bulk with the sonic hand piece device, composite was sculpted with and adapted to the cavosurface angle. Then, the composite resin was cured for $20 \mathrm{sec}$. (Bluephase N Ivoclar Vivadent). The light tip was placed as close as possible to the restoration.

Group III: Before composite application, preparation and bonding procedure was done exactly as it was in group I and II. Tetric N-Ceram was applied using an incremental filling technique each increment didn't exceed $2 \mathrm{~mm}$ in thickness. Each increment was polymerized for $20 \mathrm{sec}$ with a LED light-curing unit.

Two experienced double-blinded operators not involved in the study accomplished the evaluation of restoration. The operators were standardized to a determined level of inter- and intra-examiner covenant of at least a Kappa value of $95 \%$ for each parameter. The restorations were assessed and evaluated at baseline, 6, 12, 18 and 24 months using modified US Public Health Service Ryge criteria and recorded as Alpha, Bravo, or Charlie (Table 3). Where, Alpha corresponds to excellent, Bravo to clinically acceptable, and Charlie to clinically unacceptable results. Postoperative sensitivity was measured by blowing a stream of air for a period of $3 \mathrm{sec}$ at a distance of 2 to $3 \mathrm{~cm}$ from the restoration and by moving the probe above the restored tooth surface. By using piece of gauze the restored tooth was isolated from the adjacent teeth ${ }^{[20]}$.

\section{Replica preparation}

Epoxy resin replicas were prepared for marginal analysis by making polysiloxane impressions (Express XT, 3M ESPE, Germany) to the restored teeth under investigation. Impressions were then poured with clear epoxy resin to produce the replica that was gold sputtered to be examined under the scanning electron microscopy (JEOL JXA-840A scanning microscope, USA) initially at magnification (12x) for the detection of all restoration margins, followed by a higher magnification $(50 \mathrm{x}-500 \mathrm{x})$ to examine the entire margin of the restoration [21]. Image $\mathrm{J}$ software (National Institutes of Health, Bethesda, MD, USA) was used; Image $\mathrm{J}$ is a Javabased image processing program developed at the national institutes of health and the laboratory for optical and computational instrumentation. The marginal integrity was measured as percentages of continuous margins to the full marginal length ${ }^{[22,23]}$. Steps of margins measurements using Image $\mathbf{J}$ software were shown in (Figure 1).

The statistical analyses were carried out with (SPSS version 22.0 software package (SPSS, Chicago, IL, USA). 
TABLE (1) The material and its composition used in the study

\begin{tabular}{|c|c|c|}
\hline Materials & Composition & Manufacturer \\
\hline $\begin{array}{l}\text { Tetric N-Ceram } \AA \\
\text { Bulk-Fill } \\
\text { composite }\end{array}$ & $\begin{array}{l}\text { The monomer matrix is composed of dimethacrylates ( } 20-21 \% \text { weight). } \\
\text { Thefillers contain barium glass, ytterbium trifluoride, mixed oxide and } \\
\text { prepolymer ( } 79-81 \% \text { weight). Additional contents: additives, catalysts, } \\
\text { stabilizers andPigments ( }<1.0 \% \text { weight). The total content of inorganic fillers is } \\
76-77 \% \text { Weight or } 53-54 \% \text { volume. The particle size of the inorganic fillers is } \\
\text { between } 40 \mathrm{~nm} \text { and } 3,000 \mathrm{~nm} \text { with a mean particle size of } 550 \mathrm{~nm} \text {. }\end{array}$ & $\begin{array}{l}\text { IvoclarVivadent, } \\
\text { Schaan, } \\
\text { Liechtenstein }\end{array}$ \\
\hline SonicFill & $\begin{array}{l}\text { Glass, oxide, chemicals } \\
\text { 3-trimethoxysilylpropyl methacrylate } \\
\text { Silicon dioxide } \\
\text { Ethoxylatedbisphenol-Adimethacrylate } \\
\text { Bisphenol-A-bis-(2-hydroxy-3-mehacryloxypropyl) ether } \\
\text { Triethyleneglycoldimethacrylate }\end{array}$ & $\begin{array}{l}\text { Kerr Corporation, } \\
\text { USA }\end{array}$ \\
\hline $\begin{array}{l}\text { Tetric } \AA \text { N-Ceram } \\
\text { Nano-hybrid } \\
\text { incremental } \\
\text { composite }\end{array}$ & $\begin{array}{l}\text { Dimethacrylates }(19-20 \mathrm{wt} \%) \text {. The fillers contain barium glass, ytterbium } \\
\text { trifluoride, mixed oxide and Copolymers }(80-81 \mathrm{wt} \%) \text {. } \\
\text { Additives, catalysts, stabilizers and pigments are additionalcontents }(<1 \mathrm{wt} \text {. } \\
\%) \text {. The total content of inorganic fillers is } 55-57 \mathrm{vol} \% \text {. The particle size of } \\
\text { inorganic fillers is between } 40 \mathrm{~nm} \text { and } 3000 \mathrm{~nm} .\end{array}$ & $\begin{array}{l}\text { IvoclarVivadent, } \\
\text { Schaan, } \\
\text { Liechtenstein }\end{array}$ \\
\hline OptiBond FL & $\begin{array}{l}\text { Etching : } 37 \% \text { phosphoric acid } \\
\text { Primer :2-hydroxyethylmethacrylate (HEMA) } \\
\text { Glycerophosphate-dimethacrylate (GPDM) (MMEP) Ethanol water - initiators } \\
\text { Bond :Bis-phenol A diglycidylmethacrylate } \\
\text { 2-hydroxyethylmethacrylate (HEMA) } \\
\text { Glycerophosphate-dimethacrylate (GPDM) Barium-aluminum borosilicate } \\
\text { glass, Disodium hexa-fluoro-silicate Fumed silica }\end{array}$ & $\begin{array}{l}\text { Kerr Corporation, } \\
\text { USA }\end{array}$ \\
\hline $\begin{array}{l}\text { Clear Epoxy resin } \\
\text { ( EPODEX) }\end{array}$ & $\begin{array}{l}\text { (A-component) : 2,2 '- [(1-methylethylidene) bis (4,1-phenyleneoxymethylene)] } \\
\text { bisoxirane Reaction mass of 2,2'-[methylenebis (4,1-phenyleneoxymethylene)] } \\
\text { dioxirane and [ } 2-(\{2-\text { [4- (oxirane-2-ylmethoxy) benzyl] phenoxy }\} \text { methyl) } \\
\text { oxiranes and [2,2 '- [methylene bis (2,1-phenyleneoxymethylene)] dioxirane } \\
\text { oxiranes, mono (C 12-14 alkyloxy) methyl) derivs. } \\
\text { B-component); Reaction product: 3-aminomethyl-3,5,5-trimethylcyclohex- } \\
\text { ylamine and 4,4'-isopropylidenediphenol, oligomeric reaction products with } \\
\text { 1-chloro-2,3-epoxypropane m-phenylenebis (methylamine) salicylic acid. }\end{array}$ & $\begin{array}{l}\text { Company EPODEX } \\
\text { North Rhine- } \\
\text { Westphalia Germany }\end{array}$ \\
\hline
\end{tabular}

TABLE (2) Distribution of tooth restored in relation to different tested groups

\begin{tabular}{|c|c|c|c|c|c|}
\hline Groups & Upper premolar & Lower premolar & Upper molar & Lower molar & Total \\
\hline Group 1 & 3 & 4 & 10 & 13 & 30 \\
\hline Group 2 & 2 & 2 & 9 & 17 & 30 \\
\hline Group 3 & 4 & 3 & 9 & 14 & 30 \\
\hline Total & 9 & 9 & 28 & 44 & 90 \\
\hline
\end{tabular}


TABLE (3): Modified United States Public Health Service evaluation criteria

\begin{tabular}{|c|c|c|c|}
\hline Criteria & \multicolumn{2}{|c|}{$\begin{array}{l}\text { Analogous USPHS } \\
\text { criteria }\end{array}$} & Description \\
\hline \multirow{3}{*}{ 1-Marginal integrity } & - Alpha & (A) & No visible crevice or so small that the probe just catch it and not fall in. \\
\hline & - Bravo & (B) & Explorer tip fall into crevice but the dentin was not exposed. \\
\hline & - Charlie & (C) & $\begin{array}{l}\text { Explorer penetrates into a crevice that is of depth that exposes dentin or } \\
\text { base. }\end{array}$ \\
\hline \multirow{3}{*}{ 2-Marginal discoloration } & - Alpha & (A) & Mismatch is outside the acceptable range \\
\hline & - Bravo & (B) & Absence of marginal discoloration \\
\hline & - Charlie & (C) & Presence of marginal discoloration, limited and not extended \\
\hline \multirow{3}{*}{ 3-Color match } & - Alpha & (A) & $\begin{array}{l}\text { The restoration matches the adjacent tooth structure in color, shade or } \\
\text { translucency }\end{array}$ \\
\hline & - Bravo & (B) & $\begin{array}{l}\text { Mismatch in color, shade or translucency between the restoration and } \\
\text { the adjacent tooth }\end{array}$ \\
\hline & - Charlie & (C) & $\begin{array}{l}\text { The mismatch in color and translucency is outside the acceptable range } \\
\text { of tooth color and translucency }\end{array}$ \\
\hline \multirow{3}{*}{ 4- Surface roughness } & -Alpha & (A) & Surface is smooth as the surrounding enamel. \\
\hline & -Bravo & (B) & Surface is rougher than surrounding enamel. \\
\hline & - Charlie & (C) & Surface is very rough. \\
\hline \multirow{3}{*}{ 5- Anatomic form (wear) } & - Alpha & (A) & Restoration is continuous with existing anatomic form. \\
\hline & - Bravo & (B) & $\begin{array}{l}\text { Restoration is discontinuous with existing anatomic form, but missing } \\
\text { material is not sufficient to expose dentin or base. }\end{array}$ \\
\hline & - Charlie & (C) & Sufficient restorative material is missing to expose dentin or base. \\
\hline \multirow[t]{3}{*}{ 6- Retention of the restoration } & - Alpha & (A) & Complete retention of the restoration. \\
\hline & - Bravo & (B) & Partial retention of the restoration. \\
\hline & - Charlie & (C) & Complete loss of the restoration. \\
\hline \multirow[t]{2}{*}{ 7- secondry caries } & - Alpha & (A) & No evidence for caries contiguous with the restoration margins. \\
\hline & - Bravo & (B) & Caries contiguous with the restoration margin. \\
\hline \multirow[t]{3}{*}{ 8- Postoperative sensitivity } & - Alpha & (A) & Not present. \\
\hline & - Bravo & (B) & Sensitive but diminishing in intensity. \\
\hline & - Charlie & (C) & Constant sensitivity, not diminishing in intensity. \\
\hline
\end{tabular}




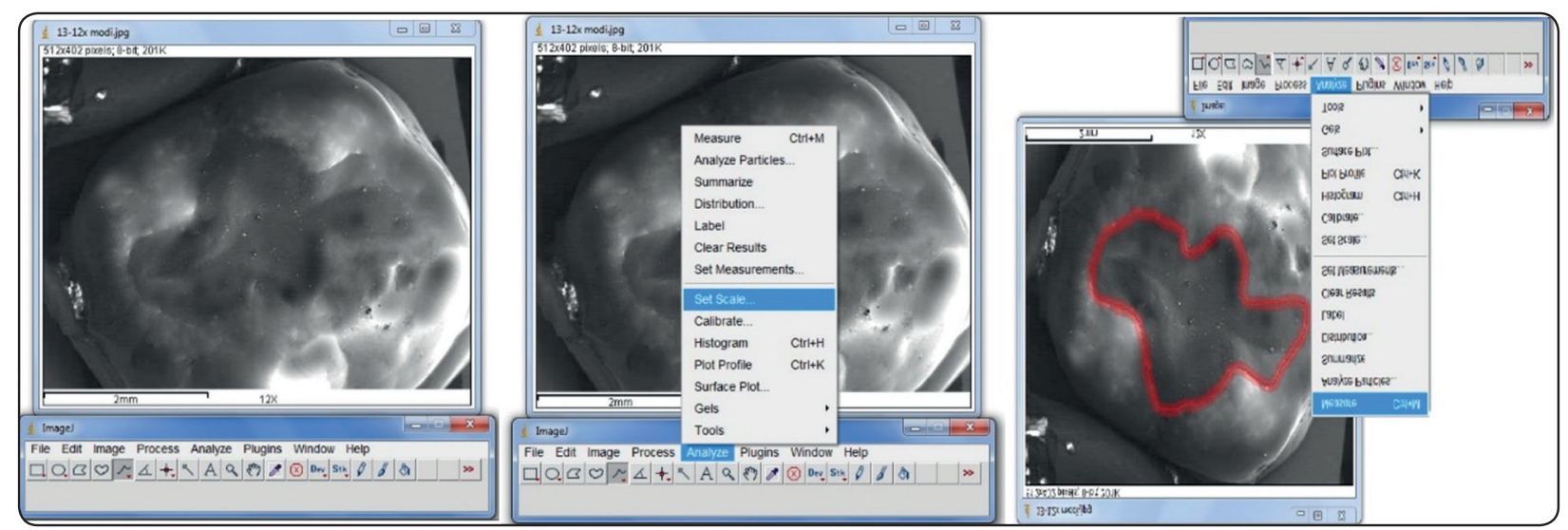

Fig. (1) Analysis of Scanning Electron Microscopy micrograph using Image J software

\section{RESULTS}

Recall rates at six, 12, 18, were $100 \%$ where at 24 month it was $93.3 \%$. The retention rate was $100 \%$.

\section{Marginal integrity:}

As shown in (Table 4) and (Figure 2) below, the marginal integrity of groups I, II and III recorded Alpha score (100\%) at base line, six and 12 months.
At 18-month follow-up period, $10 \%, 6.67 \%$ and $30 \%$ of group I, II and III, respectively recorded Bravo score. After 24 months, $3.57 \%$ and $21.43 \%$ of group I and III, respectively recorded Charlie score. $7.14 \%, 14.29 \%$ and $10.71 \%$ of group I, group II and group III, respectively recorded Bravo score. Chi square test exhibited no statistically significant difference amongst the three tested material except after 18 and 24 months follow-up (P-value=0.026) TABLE (4) Results of the marginal integrity of the tested groups at different follow up periods.

\begin{tabular}{|c|c|c|c|c|c|c|c|c|c|c|c|}
\hline \multirow{3}{*}{\multicolumn{2}{|c|}{ Marginal integrity }} & \multicolumn{8}{|c|}{ Groups } & \multirow{2}{*}{\multicolumn{2}{|c|}{ Chi-Square }} \\
\hline & & \multicolumn{2}{|c|}{ Group I } & \multicolumn{2}{|c|}{ Group II } & \multicolumn{2}{|c|}{ Group III } & \multicolumn{2}{|c|}{ Total } & & \\
\hline & & $\mathbf{N}$ & $\%$ & $\mathbf{N}$ & $\%$ & $\mathbf{N}$ & $\%$ & $\mathbf{N}$ & $\%$ & $\mathrm{X}^{2}$ & P-value \\
\hline \multirow{3}{*}{ Baseline } & Alpha & 30 & 100.00 & 30 & 100.00 & 30 & 100.00 & 90 & 100.00 & \multirow{3}{*}{ - } & \multirow{3}{*}{ - } \\
\hline & Bravo & 0 & 0.00 & 0 & 0.00 & 0 & 0.00 & 0 & 0.00 & & \\
\hline & Charlie & 0 & 0.00 & 0 & 0.00 & 0 & 0.00 & 0 & 0.00 & & \\
\hline \multirow{3}{*}{6 Months } & Alpha & 30 & 100.00 & 30 & 100.00 & 30 & 100.00 & 90 & 100.00 & \multirow{3}{*}{ - } & \multirow{3}{*}{ - } \\
\hline & Bravo & 0 & 0.00 & 0 & 0.00 & 0 & 0.00 & 0 & 0.00 & & \\
\hline & Charlie & 0 & 0.00 & 0 & 0.00 & 0 & 0.00 & 0 & 0.00 & & \\
\hline \multirow{3}{*}{12 Months } & Alpha & 30 & 100.00 & 30 & 100.00 & 30 & 100.00 & 90 & 100.00 & \multirow{3}{*}{-} & \\
\hline & Bravo & 0 & 0.00 & 0 & 0.00 & 0 & 0.00 & 0 & 0.00 & & \\
\hline & Charlie & 0 & 0.00 & 0 & 0.00 & 0 & 0.00 & 0 & 0.00 & & \\
\hline \multirow{3}{*}{18 Months } & Alpha & 27 & 90.00 & 28 & 93.33 & 21 & 70.00 & 76 & 84.44 & \multirow{3}{*}{7.274} & \multirow{3}{*}{$0.026^{*}$} \\
\hline & Bravo & 3 & 10.00 & 2 & 6.67 & 9 & 30.00 & 14 & 15.56 & & \\
\hline & Charlie & 0 & 0.00 & 0 & 0.00 & 0 & 0.00 & 0 & 0.00 & & \\
\hline \multirow{3}{*}{24 Months } & Alpha & 25 & 89.29 & 24 & 85.71 & 19 & 67.86 & 68 & 80.95 & \multirow{3}{*}{10.436} & \multirow{3}{*}{$0.034 *$} \\
\hline & Bravo & 2 & 7.14 & 4 & 14.29 & 3 & 10.71 & 9 & 10.71 & & \\
\hline & Charlie & 1 & 3.57 & 0 & 0.00 & 6 & 21.43 & 7 & 8.33 & & \\
\hline \multirow{4}{*}{ Chi-Square } & B-A6M & & - & & - & & - & & & & \\
\hline & B-A12M & & - & & - & & - & & & & \\
\hline & B-A18M & & .236 & & 472 & & 004* & & & & \\
\hline & B-A24M & & 184 & & 104 & & $03^{*}$ & & & & \\
\hline
\end{tabular}




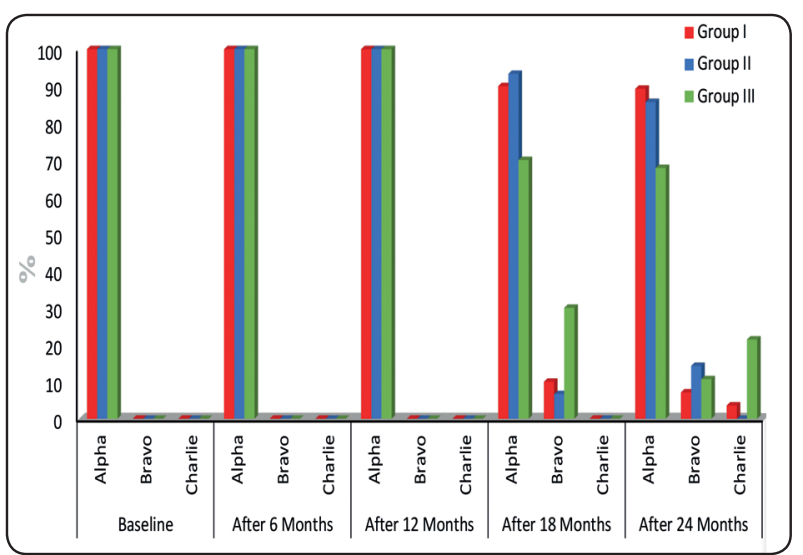

Fig. (2) Bar chart representing results of the marginal integrity of the tested groups at different follow up periods.

and (P-value=0.034), respectively. Regarding group I and II there was no statistically significant difference between different follow up periods. However, group III revealed significant difference when baseline scores were compared to that after 18 and 24 months, $(\mathrm{P}$-value $=0.004),(\mathrm{P}$-value $=0.003)$ respectively.

\section{Marginal discoloration}

(Table 5) and (Figure 3) show that none of the restorations revealed marginal discoloration in baseline, six and 12 months. However, after 18 months follow-up, $6.67 \%, 3.33 \%$ and $23.33 \%$ of the restorations of group I, group II and group III, respectively recorded Bravo score. Charlie score recorded at the end of 24 months in one restoration in group I and five restorations in group III while Bravo score was recorded for $3.57 \%, 7.14 \%$ and $14.29 \%$ of group I, group II and group III, respectively. Significant difference was detected among the three tested groups at 18 and 24 months evaluation where (P-value $=0.031)$ and ( $\mathrm{p}$-value $=0.034)$, respectively. Group I and II exhibited no significant differences along the follow-up period. On the other hand, group III showed significant difference when comparing base line scores with 18 months $(\mathrm{P}$-value $=0.016)$ and 24 months $(\mathrm{P}$-value $=0.003)$.

TABLE (5) Results of the marginal discoloration of the tested groups at different follow up periods

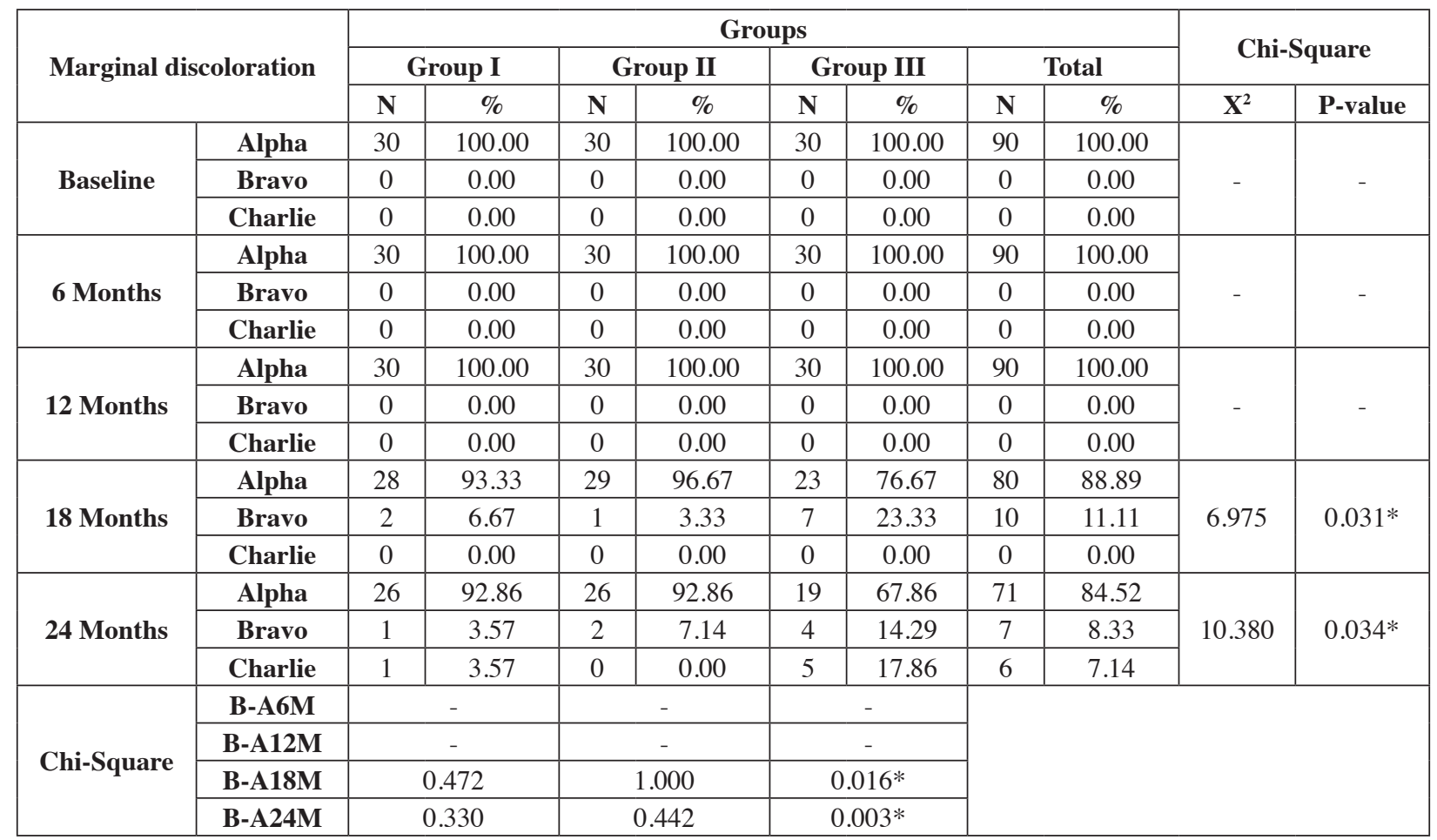




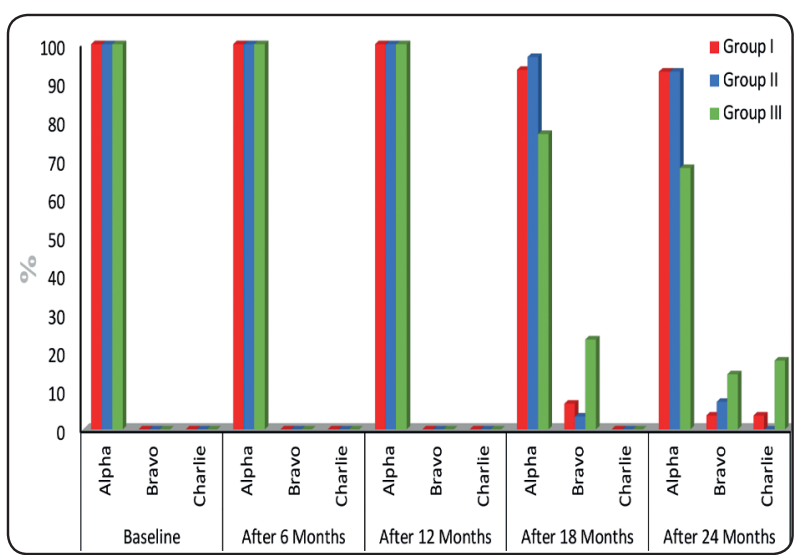

Fig. (3) Bar chart representing results of the marginal discoloration of the tested groups at different follow up periods.

\section{Color match and surface roughness:}

As shown in (Table 6) and (Figure 4), the color match of groups I and II and III recorded Alpha score $(100 \%)$ at base line and six months where at 12 months, Bravo score was recorded in $6.67 \%$ of group I only whereas at 18 months Bravo score appeared in all tested groups as $10.00 \%, 3.33 \%$ and
$20.00 \%$, respectively. At 24 months, Charlie score was observed in group I and III where it was recorded as $7.14 \%$ and $10.71 \%$, respectively. Whereas Bravo score was $14.29 \%$ in all tested groups at 24 months. Chi-square test showed no statistically significant difference among the three tested groups at any follow up period. On the other hand, the difference was statistically significance when comparing base line scores with scores at 24 months regarding group I and III where ( $\mathrm{P}-$ value $=0.028)$ and $\mathrm{P}$-value $=0.014)$, respectively. Alternatively, group II exhibited no statistically significant difference when scores obtained throughout the follow up periods were compared with baseline scores.

\section{Anatomic form (wear):}

Concerning the anatomic form (wear), group II recorded Alpha score throughout the study. On the other hand, Bravo score was $3.33 \%$ in group I and group III at 18 months and became $3.57 \%$ and 7.14\% after 24 months in group I and group III,

TABLE (6) Results of the color match and surface roughness of the tested groups at different follow up periods

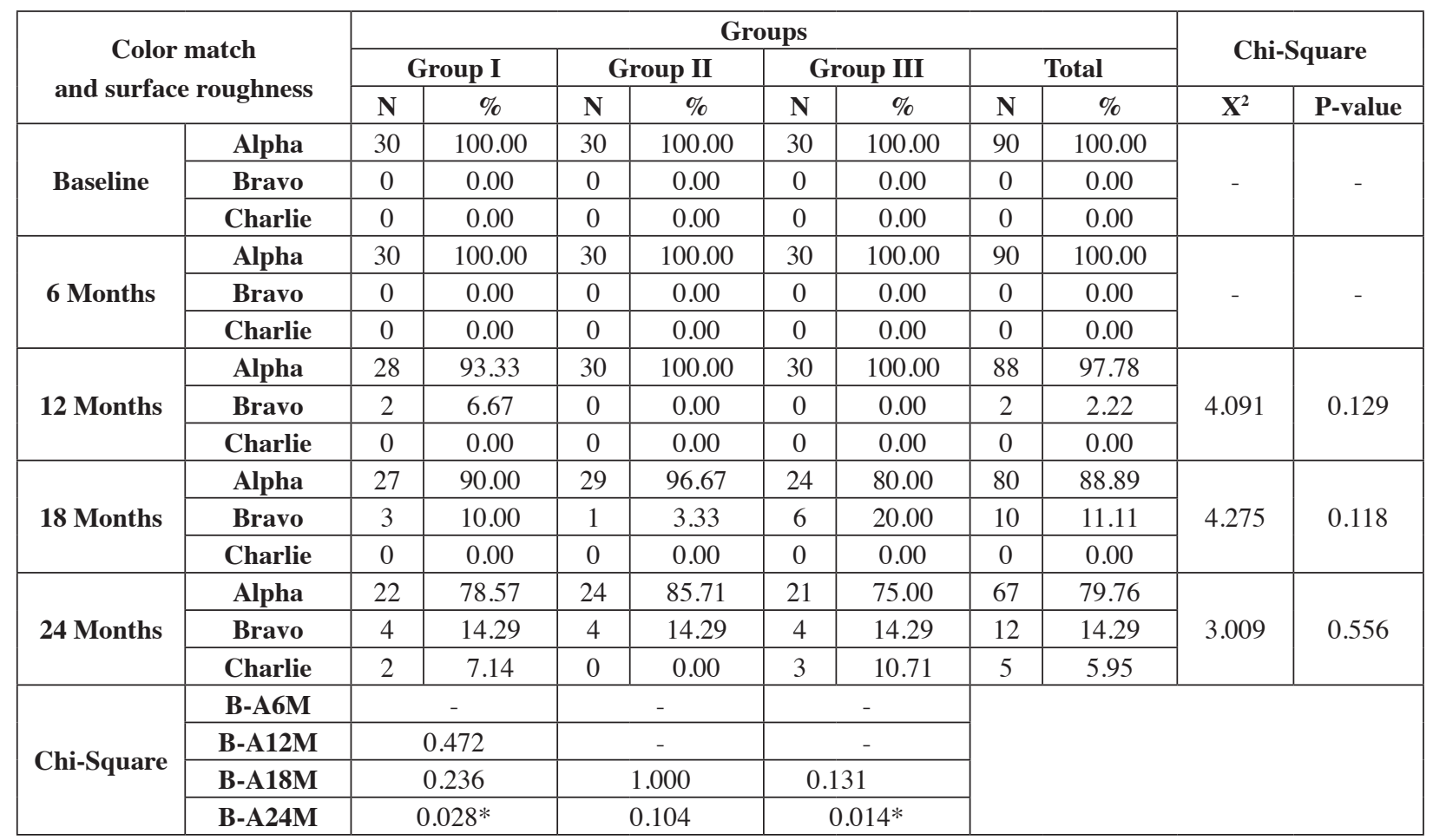




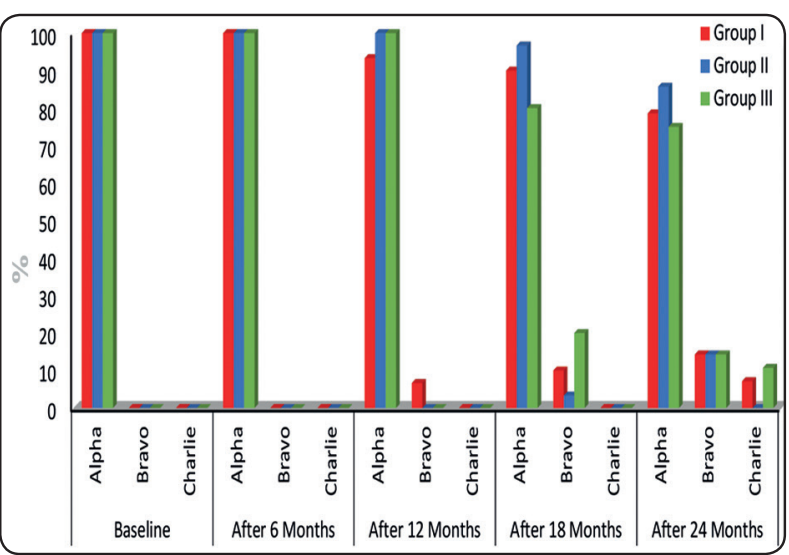

Fig. (4) Bar chart representing results of the color match and surface roughness of the tested groups at different follow up periods.

respectively. Chi-square test showed no statistically significant difference amongst the three tested groups at any follow-up period $(\mathrm{P}$-value $=0.600)$ and (P-value $=0.355)$ at 18 and 24 months, respectively. In addition, no statistically significant difference was recorded amongst the different recall periods of the tested groups as shown in (Table 7) and (Figure 5).

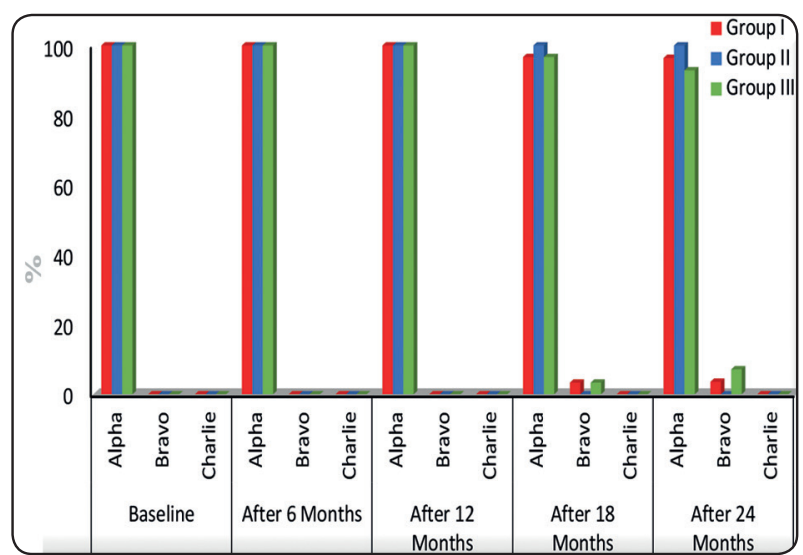

Fig. (5) Bar chart representing results of the anatomic form (wear) of the tested groups at different follow up periods.

\section{Retention of restoration:}

There were no significant differences either amongst the three tested restorative materials nor the follow up period of recall of each one regarding the retention of the restoration $(\mathrm{P}-\mathrm{value}=0.364)$ and $(\mathrm{P}-\mathrm{value}=0.129)$ at 18 and 24 months, respectively.

Bravo score of $3.33 \%$ was recorded at 18 months TABLE (7) Results of the anatomic form (wear) of the tested groups at different follow up periods

\begin{tabular}{|c|c|c|c|c|c|c|c|c|c|c|c|}
\hline \multirow{3}{*}{\multicolumn{2}{|c|}{ Anatomic form (wear) }} & \multicolumn{8}{|c|}{ Groups } & \multirow{2}{*}{\multicolumn{2}{|c|}{ Chi-Square }} \\
\hline & & \multicolumn{2}{|c|}{ Group I } & \multicolumn{2}{|c|}{ Group II } & \multicolumn{2}{|c|}{ Group III } & \multicolumn{2}{|c|}{ Total } & & \\
\hline & & $\mathbf{N}$ & $\%$ & $\mathbf{N}$ & $\%$ & $\mathbf{N}$ & $\%$ & $\mathbf{N}$ & $\%$ & $\mathbf{X}^{2}$ & P-value \\
\hline \multirow{3}{*}{ Baseline } & Alpha & 30 & 100.00 & 30 & 100.00 & 30 & 100.00 & 90 & 100.00 & \multirow{3}{*}{ - } & \multirow{3}{*}{ 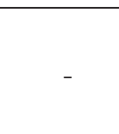 } \\
\hline & Bravo & 0 & 0.00 & 0 & 0.00 & 0 & 0.00 & 0 & 0.00 & & \\
\hline & Charlie & 0 & 0.00 & 0 & 0.00 & 0 & 0.00 & 0 & 0.00 & & \\
\hline \multirow{3}{*}{6 Months } & Alpha & 30 & 100.00 & 30 & 100.00 & 30 & 100.00 & 90 & 100.00 & \multirow{3}{*}{ - } & \multirow{3}{*}{ - } \\
\hline & Bravo & 0 & 0.00 & 0 & 0.00 & 0 & 0.00 & 0 & 0.00 & & \\
\hline & Charlie & 0 & 0.00 & 0 & 0.00 & 0 & 0.00 & 0 & 0.00 & & \\
\hline \multirow{3}{*}{12 Months } & Alpha & 30 & 100.00 & 30 & 100.00 & 30 & 100.00 & 90 & 100.00 & \multirow{3}{*}{ - } & \multirow{3}{*}{-} \\
\hline & Bravo & 0 & 0.00 & 0 & 0.00 & 0 & 0.00 & 0 & 0.00 & & \\
\hline & Charlie & 0 & 0.00 & 0 & 0.00 & 0 & 0.00 & 0 & 0.00 & & \\
\hline \multirow{3}{*}{18 Months } & Alpha & 29 & 96.67 & 30 & 100.00 & 29 & 96.67 & 88 & 97.78 & \multirow{3}{*}{1.023} & \multirow{3}{*}{0.600} \\
\hline & Bravo & 1 & 3.33 & 0 & 0.00 & 1 & 3.33 & 2 & 2.22 & & \\
\hline & Charlie & 0 & 0.00 & 0 & 0.00 & 0 & 0.00 & 0 & 0.00 & & \\
\hline \multirow{3}{*}{24 Months } & Alpha & 27 & 96.43 & 28 & 100.00 & 26 & 92.86 & 81 & 96.43 & \multirow{3}{*}{2.074} & \multirow{3}{*}{0.355} \\
\hline & Bravo & 1 & 3.57 & 0 & 0.00 & 2 & 7.14 & 3 & 3.57 & & \\
\hline & Charlie & 0 & 0.00 & 0 & 0.00 & 0 & 0.00 & 0 & 0.00 & & \\
\hline \multirow{4}{*}{ Chi-Square } & B-A6M & & - & & - & & - & & & & \\
\hline & B-A12M & & - & & - & & - & & & & \\
\hline & B-A18M & & .000 & & - & & 000 & & & & \\
\hline & B-A24M & & .972 & & - & & 442 & & & & \\
\hline
\end{tabular}


in group III and 2 restorations recorded Bravo at 24 months. Charlie was not obtained which means that all tested materials were clinically accepted during the period of study. (Table 8) and (Figure 6).

\section{Secondary caries and postoperative sensitivity}

There were no detected secondary caries and none of the patients has been evaluated for having postoperative sensitivity with no significant difference among the tested restorative material as shown in (Table 9) and (Figure 7).

TABLE (8) Results of the retention of the tested groups at different follow up periods

\begin{tabular}{|c|c|c|c|c|c|c|c|c|c|c|c|}
\hline \multirow{3}{*}{\multicolumn{2}{|c|}{ Retention }} & \multicolumn{8}{|c|}{ Groups } & \multirow{2}{*}{\multicolumn{2}{|c|}{ Chi-Square }} \\
\hline & & \multicolumn{2}{|c|}{ Group I } & \multicolumn{2}{|c|}{ Group II } & \multicolumn{2}{|c|}{ Group III } & \multicolumn{2}{|c|}{ Total } & & \\
\hline & & $\mathbf{N}$ & $\%$ & $\mathbf{N}$ & $\%$ & $\mathbf{N}$ & $\%$ & $\mathbf{N}$ & $\%$ & $\mathbf{X}^{2}$ & P-value \\
\hline \multirow{3}{*}{ Baseline } & Alpha & 30 & 100.00 & 30 & 100.00 & 30 & 100.00 & 90 & 100.00 & \multirow{3}{*}{ - } & \multirow{3}{*}{ - } \\
\hline & Bravo & 0 & 0.00 & 0 & 0.00 & 0 & 0.00 & 0 & 0.00 & & \\
\hline & Charlie & 0 & 0.00 & 0 & 0.00 & 0 & 0.00 & 0 & 0.00 & & \\
\hline \multirow{3}{*}{6 Months } & Alpha & 30 & 100.00 & 30 & 100.00 & 30 & 100.00 & 90 & 100.00 & \multirow{3}{*}{ - } & \multirow{3}{*}{ - } \\
\hline & Bravo & 0 & 0.00 & 0 & 0.00 & 0 & 0.00 & 0 & 0.00 & & \\
\hline & Charlie & 0 & 0.00 & 0 & 0.00 & 0 & 0.00 & 0 & 0.00 & & \\
\hline \multirow{3}{*}{12 Months } & Alpha & 30 & 100.00 & 30 & 100.00 & 30 & 100.00 & 90 & 100.00 & \multirow{3}{*}{ - } & \multirow{3}{*}{ - } \\
\hline & Bravo & 0 & 0.00 & 0 & 0.00 & 0 & 0.00 & 0 & 0.00 & & \\
\hline & Charlie & 0 & 0.00 & 0 & 0.00 & 0 & 0.00 & 0 & 0.00 & & \\
\hline \multirow{3}{*}{18 Months } & Alpha & 30 & 100.00 & 30 & 100.00 & 29 & 96.67 & 89 & 98.89 & \multirow{3}{*}{2.022} & \multirow{3}{*}{0.364} \\
\hline & Bravo & 0 & 0.00 & 0 & 0.00 & 1 & 3.33 & 1 & 1.11 & & \\
\hline & Charlie & 0 & 0.00 & 0 & 0.00 & 0 & 0.00 & 0 & 0.00 & & \\
\hline \multirow{3}{*}{24 Months } & Alpha & 28 & 100.00 & 28 & 100.00 & 26 & 92.86 & 82 & 97.62 & \multirow{3}{*}{4.098} & \multirow{3}{*}{0.129} \\
\hline & Bravo & 0 & 0.00 & 0 & 0.00 & 2 & 7.14 & 2 & 2.38 & & \\
\hline & Charlie & 0 & 0.00 & 0 & 0.00 & 0 & 0.00 & 0 & 0.00 & & \\
\hline \multirow{4}{*}{ Chi-Square } & B-A6M & & - & & - & & - & & & & \\
\hline & B-A12M & & $\begin{array}{c}- \\
-\end{array}$ & & - & & - & & & & \\
\hline & B-A18M & & - & & - & & .000 & & & & \\
\hline & B-A24M & & - & & - & & .442 & & & & \\
\hline
\end{tabular}

TABLE (9) Results of the secondary caries and postoperative sensitivity of the tested groups at different follow up period

\begin{tabular}{|c|c|c|c|c|c|c|c|c|c|c|c|}
\hline \multirow{3}{*}{\multicolumn{2}{|c|}{$\begin{array}{l}\text { Secondary caries } \\
\text { and hypersensitivity }\end{array}$}} & \multicolumn{8}{|c|}{ Groups } & \multirow{2}{*}{\multicolumn{2}{|c|}{ Chi-Square }} \\
\hline & & \multicolumn{2}{|c|}{ Group I } & \multicolumn{2}{|c|}{ Group II } & \multicolumn{2}{|c|}{ Group III } & \multicolumn{2}{|c|}{ Total } & & \\
\hline & & $\mathbf{N}$ & $\%$ & $\mathbf{N}$ & $\%$ & $\mathbf{N}$ & $\%$ & $\mathbf{N}$ & $\%$ & $\mathbf{X}^{2}$ & P-value \\
\hline \multirow{3}{*}{ Baseline } & Alpha & 30 & 100.00 & 30 & 100.00 & 30 & 100.00 & 90 & 100.00 & \multirow{3}{*}{-} & \multirow{3}{*}{ 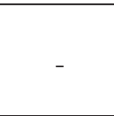 } \\
\hline & Bravo & 0 & 0.00 & 0 & 0.00 & 0 & 0.00 & 0 & 0.00 & & \\
\hline & Charlie & 0 & 0.00 & 0 & 0.00 & 0 & 0.00 & 0 & 0.00 & & \\
\hline \multirow{3}{*}{6 Months } & Alpha & 30 & 100.00 & 30 & 100.00 & 30 & 100.00 & 90 & 100.00 & \multirow{3}{*}{-} & \multirow{3}{*}{ - } \\
\hline & Bravo & 0 & 0.00 & 0 & 0.00 & 0 & 0.00 & 0 & 0.00 & & \\
\hline & Charlie & 0 & 0.00 & 0 & 0.00 & 0 & 0.00 & 0 & 0.00 & & \\
\hline \multirow{3}{*}{12 Months } & Alpha & 30 & 100.00 & 30 & 100.00 & 30 & 100.00 & 90 & 100.00 & \multirow{3}{*}{-} & \multirow{3}{*}{ - } \\
\hline & Bravo & 0 & 0.00 & 0 & 0.00 & 0 & 0.00 & 0 & 0.00 & & \\
\hline & Charlie & 0 & 0.00 & 0 & 0.00 & 0 & 0.00 & 0 & 0.00 & & \\
\hline \multirow{3}{*}{18 Months } & Alpha & 30 & 100.00 & 30 & 100.00 & 30 & 100.00 & 90 & 100.00 & \multirow{3}{*}{ - } & \multirow{3}{*}{ - } \\
\hline & Bravo & 0 & 0.00 & 0 & 0.00 & 0.00 & 0.00 & 0 & 0.00 & & \\
\hline & Charlie & 0 & 0.00 & 0 & 0.00 & 0 & 0.00 & 0 & 0.00 & & \\
\hline \multirow{3}{*}{24 Months } & Alpha & 28 & 100.00 & 28 & 100.00 & 28 & 100.00 & 84 & 97.62 & \multirow{3}{*}{ - } & \multirow{3}{*}{ - } \\
\hline & Bravo & 0 & 0.00 & 0 & 0.00 & 0 & 0.00 & 0 & 0.00 & & \\
\hline & Charlie & 0 & 0.00 & 0 & 0.00 & 0 & 0.00 & 0 & 0.00 & & \\
\hline \multirow{4}{*}{ Chi-Square } & B-A6M & & - & & - & & 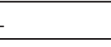 & & & & \\
\hline & B-A12M & & - & & - & & - & & & & \\
\hline & B-A18M & & - & & - & & - & & & & \\
\hline & B-A24M & & - & & - & & - & & & & \\
\hline
\end{tabular}




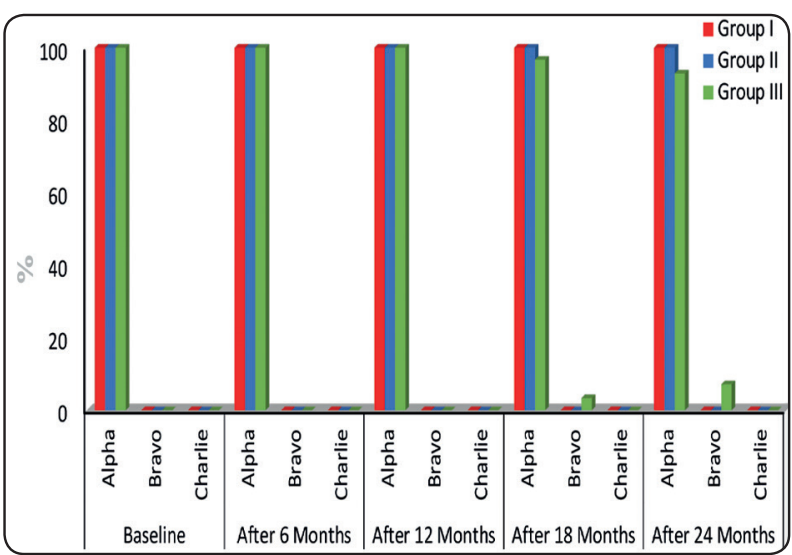

Fig. (6) Bar chart representing results of the retention of the restoration of the tested groups at different follow up periods.

Electron Microscopy Scanning for the Evaluation of Marginal Integrity

To confirm the marginal integrity results, epoxy resin replicas were performed for a tested restoration at different follow up period. These replicas were examined using Scanning Electron Microscopy (SEM). The marginal integrity was measured as percentages of continuous margins to the full marginal length.

Table 10: Mean values of perfect margin percentage of replica scanning electron microscopy evaluation results and standard deviation

\begin{tabular}{|c|c|c|c|c|c|c|c|c|c|c|c|}
\hline \multirow{2}{*}{\multicolumn{2}{|c|}{ Marginal integrity }} & \multicolumn{5}{|c|}{ Groups } & \multicolumn{2}{|c|}{ ANOVA } & \multicolumn{3}{|c|}{ TUKEY'S Test } \\
\hline & & \multicolumn{2}{|c|}{ Group I } & \multicolumn{2}{|c|}{ Group II } & Group III & \multirow{3}{*}{$\begin{array}{c}\mathbf{F} \\
1.570\end{array}$} & \multirow{3}{*}{$\begin{array}{c}\text { P-value } \\
0.214\end{array}$} & \multirow{3}{*}{ I\&II } & \multirow[t]{3}{*}{ I\&III } & \multirow[t]{3}{*}{ II\&III } \\
\hline \multirow{2}{*}{ Base line } & Range & 98.4 & $\begin{array}{l}-\quad 98.9 \\
\end{array}$ & 98.6 & 100 & $98.4 \quad-$ & & & & & \\
\hline & Mean \pm SD & 98.743 & \pm 0.141 & 98.817 & \pm 0.238 & $98.747 \pm 0.148$ & & & & & \\
\hline \multirow{2}{*}{6 Months } & Range & 98.39 & - 98.89 & 98.59 & $\begin{array}{l}-\quad 99.99 \\
\end{array}$ & $98.39 \quad-$ & \multirow{2}{*}{1.904} & \multirow{2}{*}{0.155} & & & \\
\hline & Mean \pm SD & 98.732 & \pm 0.141 & 98.810 & $\pm \quad 0.233$ & $98.729 \pm 0.160$ & & & & & \\
\hline \multirow{2}{*}{$\begin{array}{c}12 \\
\text { Months }\end{array}$} & Range & 98.39 & $\begin{array}{l}-98.89 \\
\end{array}$ & 98.6 & $\begin{array}{l}-\quad 98.8 \\
\end{array}$ & $98.38 \quad-$ & \multirow{2}{*}{0.442} & \multirow{2}{*}{0.644} & & & \\
\hline & Mean \pm SD & 98.726 & \pm 0.139 & 98.753 & $\pm \quad 0.059$ & $98.727 \pm 0.162$ & & & & & \\
\hline \multirow{2}{*}{$\begin{array}{c}18 \\
\text { Months } \\
\end{array}$} & Range & 98.34 & - 98.89 & 98.6 & $\begin{array}{l}-\quad 98.8 \\
\end{array}$ & $80.3-90.3$ & \multirow{2}{*}{733.635} & \multirow{2}{*}{$<0.001 *$} & \multirow{2}{*}{0.997} & \multirow{2}{*}{$<0.001^{*}$} & \multirow{2}{*}{$<0.001^{*}$} \\
\hline & Mean \pm SD & 98.721 & \pm 0.156 & 98.740 & $\pm \quad 0.058$ & $89.500 \pm 1.859$ & & & & & \\
\hline \multirow{2}{*}{$\begin{array}{c}24 \\
\text { Months }\end{array}$} & Range & 98.34 & $\begin{array}{l}-98.89 \\
\end{array}$ & 98.6 & 98.8 & 85.4 & \multirow{2}{*}{100.158} & \multirow{2}{*}{$<0.001^{*}$} & \multirow{2}{*}{1.000} & \multirow{2}{*}{$<0.001 *$} & \multirow{2}{*}{$<0.001 *$} \\
\hline & Mean \pm SD & 98.703 & \pm 0.100 & 98.740 & $\pm \quad 0.055$ & $77.397 \pm 11.670$ & & & & & \\
\hline \multirow{2}{*}{ B-A6M } & Differences & 0.011 & \pm 0.053 & 0.007 & $\pm \quad 0.027$ & $0.018 \pm 0.054$ & & & & & \\
\hline & Paired Test & \multicolumn{2}{|c|}{0.262} & & 190 & 0.077 & & & & & \\
\hline & Differences & 0.017 & \pm 0.053 & 0.063 & $\pm \quad 0.227$ & $0.020 \pm 0.069$ & & & & & \\
\hline В-A12M & Paired Test & 0.0 & 89 & & 137 & 0.122 & & & & & \\
\hline & Differences & 0.022 & \pm 0.128 & 0.077 & \pm 0.226 & $9.247 \pm 1.880$ & & & & & \\
\hline B-A18M & Paired Test & 0.3 & 47 & & 073 & $<0.001^{*}$ & & & & & \\
\hline & Differences & 0.041 & \pm 0.163 & 0.077 & $\pm \quad 0.225$ & $21.350 \pm 11.640$ & & & & & \\
\hline B-A24M & Paired Test & 0.1 & 83 & & 071 & $<0.001 *$ & & & & & \\
\hline
\end{tabular}

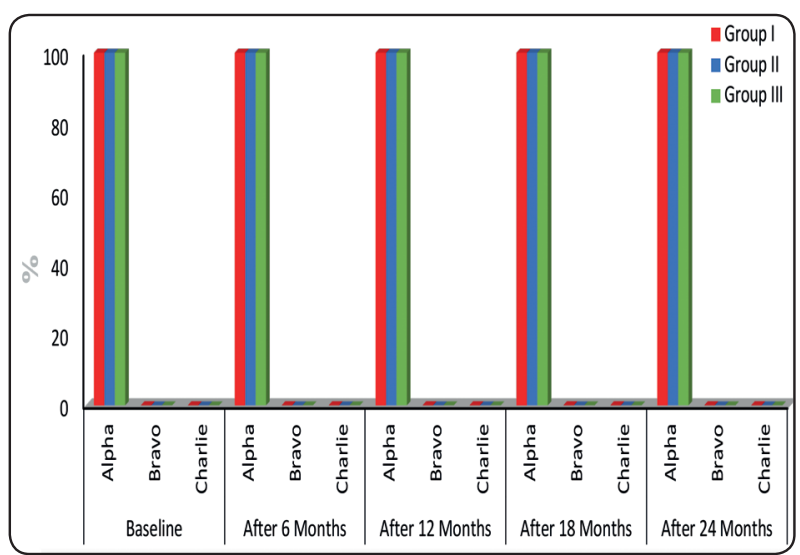

Fig. (7) Bar chart representing results of the retention of the restoration of the tested groups at different follow up periods.

As shown in (Table 10) and (Figure 8\&9) the results of clinical examination were confirmed by that of Scanning Electron Microscopy analysis of the marginal integrity where there was a significant difference among the tested groups as revealed by ANOVA test at 18 months and 24 months $(\mathrm{P}<0.001)$. TUKEY'S test exhibited the difference was between group I \& group III and also between II\&III. In addition Paired test presented no significant difference among follow up periods 
of group I and group II. On the other hand the difference was recorded significantly in group III when the comparison was made between the results recorded at baseline and the other at 18 months and 24 months $\mathrm{P}<0.001$.

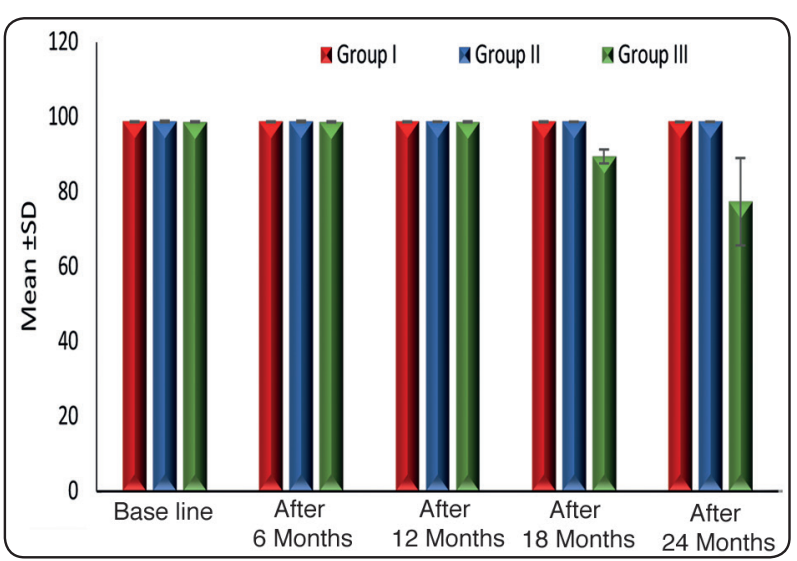

Fig. (8) Bar chart representing values of perfect margin percentage of replica scanning electron microscopy evaluation results.
Representative Scanning Electron Microscopy micrographs of restorative margins are shown in (Figure 10) represents all the restoration margins $(15 \mathrm{X})$, (Figure 11) represents margins with gap and (Figure 12) margins without gap.

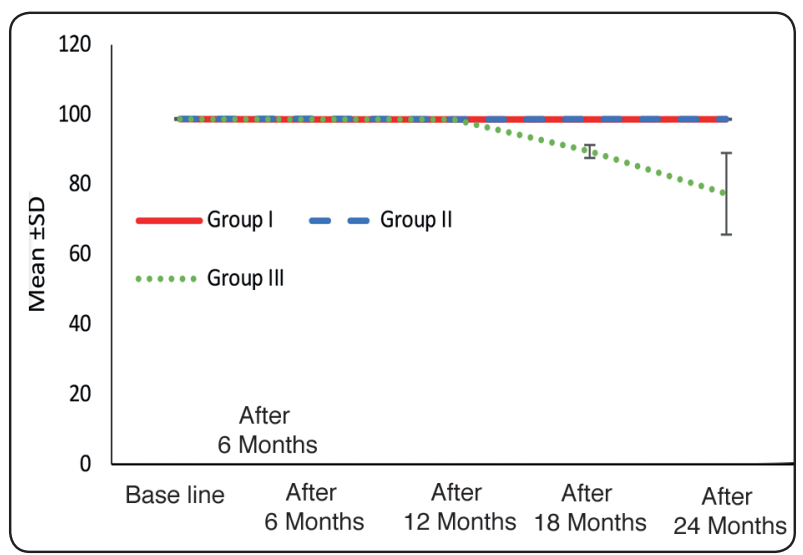

Fig. (9) Linear fit for perfect margin percentage (\%) and follow up periods for all tested materials.

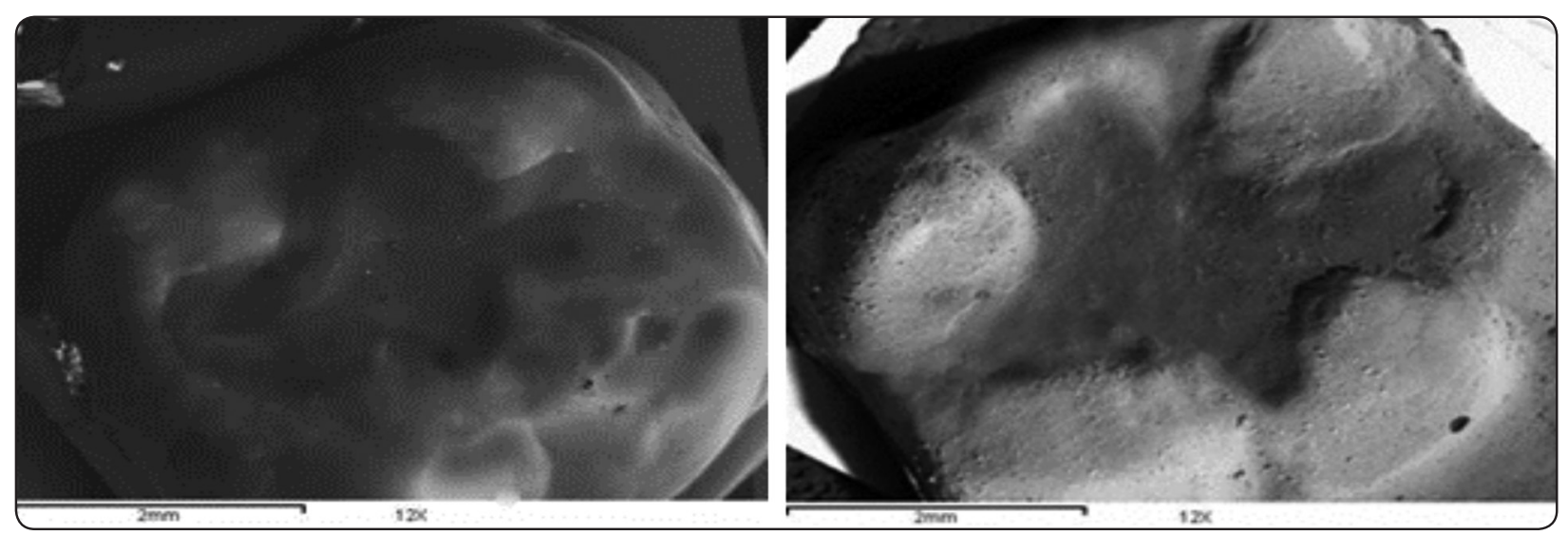

Fig. (10) SEM micrograph $(15 \mathrm{X})$ represents all the restoration margins.

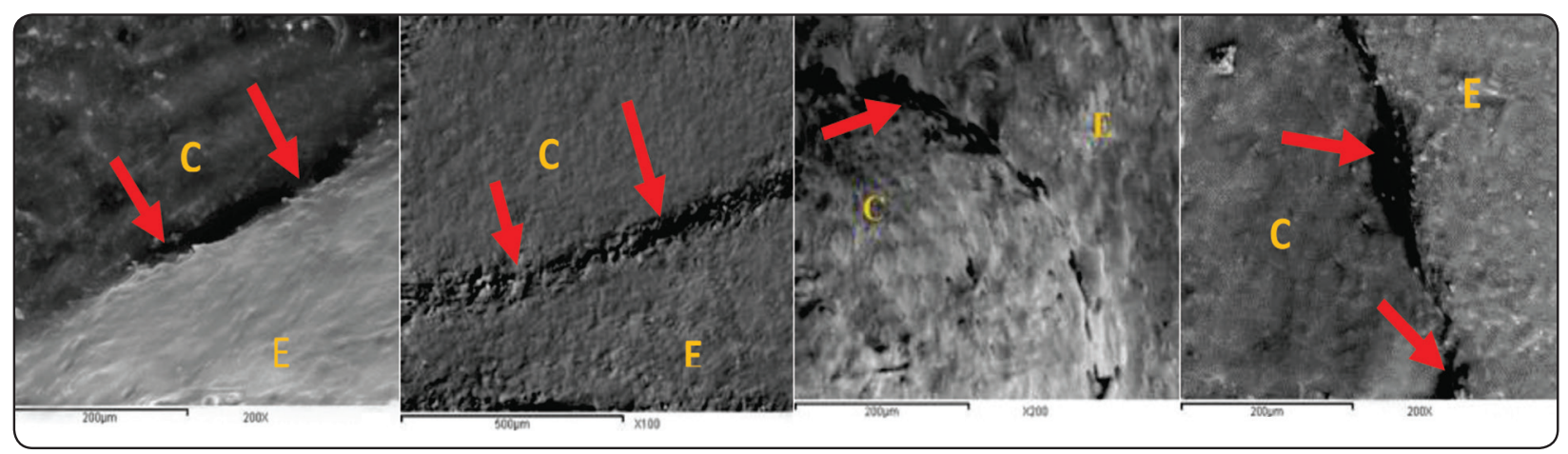

Fig. (11) Representative SEM micrograph (100 X or $200 \mathrm{X})$ of a non-continuous margin at the occlusal surface (arrows). $\mathrm{C}=$ Composite \& $\mathrm{E}=$ Enamel. 


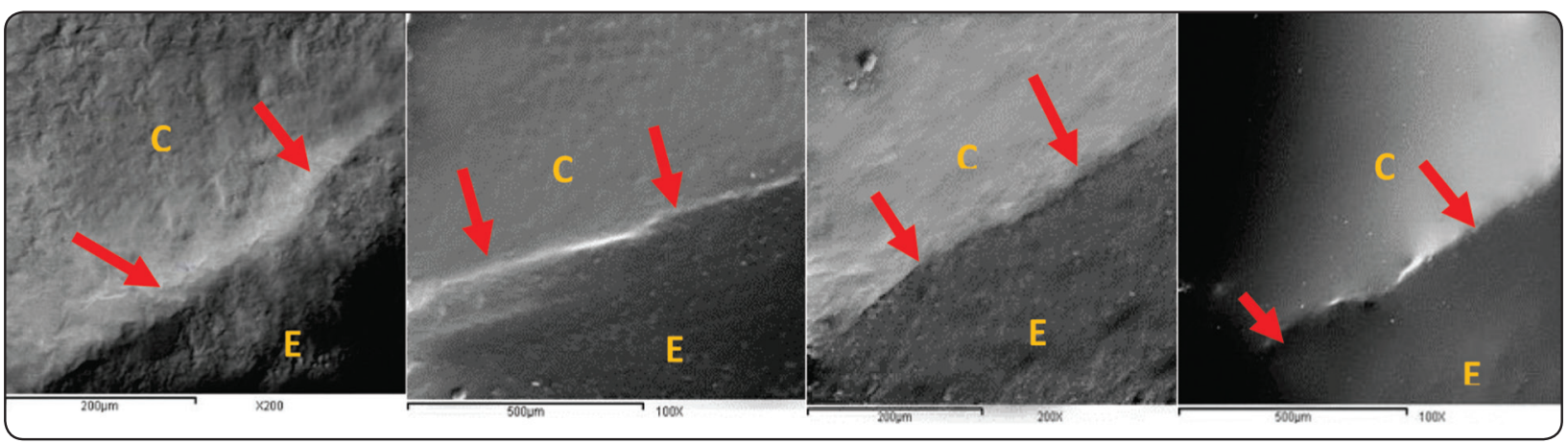

Fig. (12) Representative SEM micrograph (100 X or $200 \mathrm{X}$ ) of a continuous margin at the occlusal surface (arrows). C=Composite $\& \mathrm{E}=$ Enamel .

\section{DISCUSSION}

Composite resin restoration has been extensively used in dental practice since 1950s ${ }^{[24]}$. Composite resin has good aesthetic properties as it bonds to tooth surface via adhesive systems and thus it is more conservative than other restoration techniques ${ }^{[25]}$. Improved patient acceptance to highly aesthetic, biocompatible, economical, and clinically long-lasting restorations led to enhancement in its efficacy and durability ${ }^{[26]}$. Success of direct composite restoration depends up on several factors including: properties of the material, design of cavity preparation, and material application technique ${ }^{[27]}$. Different placement techniques of composite resin have been recommended to advance the clinical success of restoration and to overcome the side effects of polymerization shrinkage and its associated stress ${ }^{[28]}$. Numerous techniques such as indirect placement of resin restorations, controlling the curing light intensity, use of a flowable resin liner and usage of incremental layering techniques have been used to diminish the shrinkage stress effect and in this manner decrease gap formation ${ }^{[29]}$. Conversely, polymerization shrinkage and its stress effects cannot be totally avoided. Incremental technique involves placing resin composite in several increments, with each increment thickness not more than $2 \mathrm{~mm}$. This leads to satisfactory light diffusion and dispersal throughout the resin based composite material ${ }^{[30]}$. Furthermore, it declines and decreases the [C-factor] which is the ratio of bonded surface to unbonded free surface. However, the former technique has some drawbacks, such as increased chair time and inclusion of voids between the increment layers ${ }^{[31]}$. Another study also showed that the incremental technique is considered one of the methods to diminish the polymerization shrinkage techniques. However, it exhibited several problems such as presence of voids between the increment and thus increasing the probability of contamination between the layers ${ }^{[32]}$.

Several efforts were done to make the procedures of resin application and restorations easier. BulkFill technique was used to fill deep cavities all at once ${ }^{[33]}$. Contrasting to incremental filling technique, Bulk-Fill composite resin is envisioned to be inserted in increments of $4 \mathrm{~mm}$ or more ${ }^{[34]}$. The Bulk-Fill technique is less time consuming, easier and the voids are less ${ }^{[35]}$. SonicFill composite is a single-step tooth colored restorative material, which has the benefits of conventional and flowable composite resins. Bulk-Fill and nanohybrid resin composites offered comparable physical properties according to previously published scientific studies [36]. The SonicFill composite exhibited superior depth of polymerization among the tested materials using Vickers hardness measurement ${ }^{[37]}$. However, another scientific study stated that although depth of polymerization of SonicFill was not significantly different when compared to a traditional 
flowable composite resin but it presented minor polymerization shrinkage ${ }^{[33]}$.

Pre-heating of resin composites is used to advance the material handling properties. Increasing the temperature of composites prior to curing generally diminishs its viscosity, which leads to superior marginal adaptation and decreases microleakage at tooth restoration interface as it improves wetting of cavity walls ${ }^{[38]}$. In addition to this, degree of conversion is increased as a result of enhancement of radical and monomer mobility ${ }^{[39]}$. Several physical properties were also improved due to pre- heating as higher flexural, diametral tensile strength and surface hardness ${ }^{[40]}$. There are many previous studies who evaluated the effect of preheating of composite resins ${ }^{[6,41-44]}$. Despite the significant results of the former in-vitro studies, clinical evaluation and follow up are essential to expect the durability and longevity of a restorative material in the multifactorial and challenged oral conditions.

The incremental layering technique of composite application is the standard method to attain accepted clinical and physical results of restoration. It was concluded that, incremental technique led to sufficient conversion ${ }^{[45]}$. Class I cavity preparation was selected in the current study because it displays high $\mathrm{C}$ factor which is important in order to evaluate the tested material either pre-heated composite, SonicFill or incremental fill.

Pre-heated composites are convoyed by greater volumetric shrinkage leading to shrinkage stress [46]. Polymerization shrinkage stress increases in high $\mathrm{C}$ factor cavities as class 1 preparations since bonding of adhesive systems to cavity walls led to critical clinical problems such as enamel fracture, post-operative sensitivity, cuspal deflection and debonding with subsequent complications as leakage and bacterial invasion ${ }^{[47]}$.

It is important to evaluate the clinical performance of SonicFill in cavities which display high $\mathrm{C}$ factor because earlier study stated that, SonicFill presented depth of polymerization nearly $5 \mathrm{~mm}$ and recorded the highest degree of monomer conversion among the tested materials which is usually accompanied by high polymerization shrinkage stress ${ }^{[48]}$.

In the current study, the modified USPHS criteria were used as a method of clinical evaluation. It is considered a long established and commonly used method for assessment of the direct restoration regarding different parameters. The restorations in this study were carried out under similar conditions by one operator, which greatly guarantees the accuracy of the results ${ }^{[49]}$. To confirm the scores of clinical evaluation concerning marginal integrity, replica technique was used together with the modified USPHS. Examination of the margins was done using Electron Microscopy which is a reliable, efficient and non-invasive technique ${ }^{[50]}$.

SonicFill (group II) presented superior marginal adaptation regarding clinical and scanning electron microscopy evaluation since there was no Charlie score recorded at any follow up period followed by pre heated composite (group I) then incremental nano-hybrid composite (group III). Marginal adaptation shows a critical role in the success of the restoration clinically and its longevity. The purpose for improved marginal adaptation detected in SonicFill composite may be interrelated to adhesive system utilized in addition to the improved viscoelastic properties ${ }^{[51]}$. KaVo handpiece used with SonicFill depends upon sonic activation leading to a decrease in the viscosity of the material which rapidly adheres and adapt to the walls of the cavity ${ }^{[52]}$. Optibond was the adhesive system used in the current study. It is a highly filled etchand-rinse adhesive system which is considered as a gold standard technique of adhesion ${ }^{[53]}$. The same adhesive system was used with all tested restorative materials for the results to be composite type dependent. The results came in coincidence with the in-vitro study conducted by Shahidi et al., where SonicFill showed the best cervical marginal 
adaptation ${ }^{[54]}$. Tetric N-Ceram Bulk-Fill composite used in the present study and was preheated at $54^{\circ} \mathrm{C}$ and exhibited better marginal adaptation in comparison with conventional incremental fill composite resin but with no significant difference. Preheating of composite resin is a well-known way to rise the degree of flowability and decrease stickiness. It is particularly suitable for resinous material with a lot of inorganic filler particles. Concerning marginal adaptation, it has been shown that preheating guarantees better adaptation to cavity walls and margins ${ }^{[44]}$.

Current results revealed significant difference between Bulk-Fill(SonicFill) and incremental fill composite concerning marginal discoloration and marginal adaptation at 18- months which matches with the results of the study conducted by Yazici et al. where the significant differences were not obtained until 18- months ${ }^{[55]}$. On other hand, our results did not coincide with the results of the study conducted by van Dijken and Pallesen ${ }^{[56]}$. This may be attributed to the type of Bulk-Fill composite used. In the former study, flowable Bulk-Fill composites were compared with incremental fill where in the present study SonicFill composite was used without underlying flowable composite resin as one BulkFill restoration.In addition, the short-term clinical study carried out by Bayraktar et al. concluded that the Bulk-Fill restorations showed similar clinical evaluation compared to incremental fill composite resin. The divergence of results might be related to the duration of the study where the findings were attained at the end 12 months ${ }^{[57]}$. This confirms the importance of long follow-up periods of the clinical performance of restorations. The results may exhibit marked changes after longer follow-up periods. The in vitro study conducted by Gamarra et al. ${ }^{[58]}$, determined that SonicFill composite resin and the OptiBond FL adhesive system used in the study exhibited microleakage and non-continuous margins with regards to cervical margins in the dentine. The location of non-continuous margins might be the reason of the alteration between the results of the current study and this previous in-vitro study. The current study came in divergent with the study conducted by Haak et al ${ }^{[59]}$, where SonicFill composite resin and the OptiBond FL adhesive system attained an internal and marginal adaptation comparable to incremental fill composite resin. The study correlated the quality of internal and marginal adaptation to the adhesive system used.

The obtained results came in agreement with many studies, which demonstrated the effect of preheating of the composite resins. Preheating decreases the viscosity of the composite and leads to enhancement in its adherence to the walls of the prepared cavity ${ }^{[60-63]}$. Regarding surface roughness and color match, there was no significant difference between the tested materials. However SonicFill revealed relatively superior findings and lower surface roughness values. The results came in divergence with an in-vitro study which concluded that nano-hybrid Bulk-Fill composite displayed the least increase in roughness after simulated tooth brushing compared to SonicFill composite.

Group I and group III exhibited significant difference relating to surface roughness and color match when the scores of baseline were compared to that at 24 months. These findings indicate that the preheating didn't improve the surface roughness all the time of the study. These outcomes go in line with the in-vitro study carried out by Liliany and Violetta where there was a significant alteration of surface roughness of preheated composite resin compared to non-pre-heated. The previous study explains the absence of apparent effect of preheating as the reduction in temperature of composite material occurred quickly after removal from the heater ${ }^{[64]}$.

All tested groups (preheated Bulk-Fill ,SonicFill and incremental fill) exhibited no significant difference regarding the color match but these results did not go in line with the results of an invitro study where Bulk-Fill composite resins showed lower 
susceptibility to staining when immersed in tea, coffee and berry juice compared to conventional composite ${ }^{[65]}$. Another clinical study demonstrated that color match started to exhibit some change within the first 6 months which was not reported in our current study where Charlie score was recorded at 24 months follow up only. The difference in the obtained results might be related to the difference of restored cavity sites and patient hygiene ${ }^{[66]}$. Talukder et al. ${ }^{[67]}$ showed that the color mismatch caused was a result of gradual discoloration of the monomer's composite material resulting in bulk discoloration. Discoloration occurred due to external factors such as (absorption of pigment from exogenous sources in oral cavity, improper oral hygiene, smoking and dietary intake). Patients in our study adhered to all instructions given to them such as avoiding the dietary coloring staffs, smoking habits and maintained good oral hygiene. This may explain the absence of Charlie score until 24 months.

In the current study, the results of surface roughness is associated with color match change and this association is reported in many other studies [68-72]. With regards to the anatomic form (wear), there was no significant difference either between the tested groups or during the different followup periods. However, SonicFill revealed relatively superior findings. This result goes in line with the results of another scientific study which attributed this to the high microhardness of SonicFill due to the high amount of inorganic fillers present which allows the composite resin to bear functional stress ${ }^{[73]}$. Preheating leads to increased hardness and thus the composite exhibits higher resistance to wear ${ }^{[74]}$. Preheated resin composites show reduction of viscosity and improved polymerization efficacy. Heating the resin composites preceding the placement of composite and directly lightcuring rises the degree of monomer conversion rate and accordingly curing time will be reduced. Enhancement of the degree of polymerization of composite resin enhances its mechanical properties and increases its wear resistance ${ }^{[75]}$.

Regarding the retention of the restorations, Charlie score was not recorded in any of the groups with no significant difference among them. The longevity of restoration is dependent upon several factors including experience of the operator in applying the technique, physical properties of the restorative materials, use of proper isolation, the site and size of the restoration, parafunctional habits and oral hygiene of the patient. So, the results of the retention of restoration might be attributed to the use of one operator who carried out all restorations as well as proper patient selection. Moreover, the use of the same bonding agent and the same bonding procedure under proper isolation played an important role in retention of restorations. The results came in agreement with several clinical studies despite the difference in the types of BulkFill composite used, the restored cavity size and location in addition to the duration of follow-up period ${ }^{[76,77]}$. None of the patients in the current study showed neither secondary caries nor postoperative hypersensitivity during different follow up periods. Overall, all groups exhibited excellent performance in terms of secondary caries and postoperative sensitivity, and no significant differences were observed between groups. The lack of sensitivity could be the result of a liner used in deep cavities. The use of liners protects the pulpal-dentin complex, avoiding or decreasing the opportunity of thermal/electric stimuli, minimizing hydrodynamic fluid movements and encouraging the formation of reparative dentin formation. Moreover, following the biological principles in cavity preparation seized the postoperative hypersensitivity. The possible causes of secondary caries absence in all participants may be proper patient selection with good oral hygiene and exhibition of good marginal adaptation in all the follow-up period except at 24 months. These findings matches with the clinical study conducted by Sadeghi et al. and Byraktar et al ${ }^{[57,78]}$. 


\section{CONCLUSION}

Under the limitations of the current study, we can conclude that all the tested materials showed comparable satisfactory clinical behavior regarding the studied clinical parameters with the exception of marginal integrity. The SonicFill exhibited superior results regarding all tested parameters with significant differences regarding marginal integrity. Marginal analysis using replica technique and scanning electron microscope matched the clinical evaluation results. Finally, the significant alterations between different resins regarding the tested clinical parameters during follow-up periods was not evident until after18 months of clinical evaluation.

\section{REFERENCES}

1. G. Cazzaniga, M. Ottobelli, A. Ionescu, F. Garcia-Godoy, and E. Brambilla, "Surface properties of resin-based composite materials and biofilm formation: A review of the current literature.," Am. J. Dent., vol. 28, no. 6, pp. 311320, Dec. 2015.

2. A. F. Reis, M. Vestphal, R. C. do Amaral, J. A. Rodrigues, J.-F. Roulet, and M. G. Roscoe, "Efficiency of polymerization of bulk-fill composite resins: a systematic review.," Braz. Oral Res., vol. 31, no. suppl 1, p. e59, Aug. 2017.

3. X. Li, P. Pongprueksa, B. Van Meerbeek, and J. De Munck, "Curing profile of bulk-fill resin-based composites.," J. Dent., vol. 43, no. 6, pp. 664-672, Jun. 2015.

4. M. Abbasi, Z. Moradi, M. Mirzaei, M. J. Kharazifard, and S. Rezaei, "Polymerization Shrinkage of Five Bulk-Fill Composite Resins in Comparison with a Conventional Composite Resin.," J. Dent. (Tehran)., vol. 15, no. 6, pp. 365-374, Nov. 2018.

5. A. R. Benetti, C. Havndrup-Pedersen, D. Honoré, M. K. Pedersen, and U. Pallesen, "Bulk-fill resin composites: polymerization contraction, depth of cure, and gap formation," Oper Dent., vol. 40, no. 2, pp. 190-200, 2015.

6. T. T. Tauböck, Z. Tarle, D. Marovic, and T. Attin, "Preheating of high-viscosity bulk-fill resin composites: effects on shrinkage force and monomer conversion.," J. Dent., vol. 43, no. 11, pp. 1358-1364, Nov. 2015.

7. U. Lohbauer, S. Zinelis, C. Rahiotis, A. Petschelt, and G. Eliades, "The effect of resin composite pre-heating on monomer conversion and polymerization shrinkage.," Dent. Mater., vol. 25, no. 4, pp. 514-519, Apr. 2009.

8. J. Yang, N. Silikas, and D. C. Watts, "Pre-heating time and exposure duration: Effects on post-irradiation properties of a thermo-viscous resin-composite.," Dent. Mater., vol. 36, no. 6, pp. 787-793, Jun. 2020.

9. A. O. Karacan and P. Ozyurt, "Effect of preheated bulk-fill composite temperature on intrapulpal temperature increase in vitro.,” J. Esthet. Restor. Dent., Jun. 2019.

10. E. Lempel et al., "Effect of exposure time and pre-heating on the conversion degree of conventional, bulk-fill, fiber reinforced and polyacid-modified resin composites.," Dent. Mater., vol. 35, no. 2, pp. 217-228, Feb. 2019.

11. R. Hirata et al., "Effect of Sonic Resin Composite Delivery on Void Formation Assessed by Micro-computed Tomography.," Oper. Dent., vol. 43, no. 2, pp. 144-150, 2018.

12. R. B. W. Lima, C. C. M. Troconis, M. B. P. Moreno, F. Murillo-Gómez, and M. F. De Goes, "Depth of cure of Bulk-Fill resin composites: A systematic review.," J. Esthet. Restor. Dent. Off. Publ. Am. Acad. Esthet. Dent. ... [et al.], vol. 30, no. 6, pp. 492-501, Nov. 2018.

13. F. Gonçalves et al., "A comparative study of bulk-fill composites: degree of conversion, post-gel shrinkage and cytotoxicity.," Braz. Oral Res., vol. 32, p. e17, Mar. 2018.

14. R. J.-Y. Kim, Y.-J. Kim, N.-S. Choi, and I.-B. Lee, "Polymerization shrinkage, modulus, and shrinkage stress related to tooth-restoration interfacial debonding in bulk-fill composites.," J. Dent., vol. 43, no. 4, pp. 430-439, Apr. 2015.

15. R. R. Braga, L. C. C. Boaro, T. Kuroe, C. L. N. Azevedo, and J. M. Singer, "Influence of cavity dimensions and their derivatives (volume and ' $\mathrm{C}$ ' factor) on shrinkage stress development and microleakage of composite restorations.," Dent. Mater., vol. 22, no. 9, pp. 818-823, Sep. 2006.

16. A. Van Ende, A. Mine, J. De Munck, A. Poitevin, and B. Van Meerbeek, "Bonding of low-shrinking composites in high C-factor cavities.," J. Dent., vol. 40, no. 4, pp. 295303, Apr. 2012

17. M. A. Ghulman, "Effect of cavity configuration (C factor) on the marginal adaptation of low-shrinking composite: a comparative ex vivo study.," Int. J. Dent., vol. 2011, p. 159749, 2011.

18. K. Kaczor-Wiankowska, S. Lipa, M. Krasowski, J. Sokołowski, K. Lewusz-Butkiewicz, and A. Nowicka, "Evaluation of gap formation at the composite resin-tooth 
interface after using universal adhesives: In vitro SEM study using the replica technique," Microsc. Res. Tech., vol. 83, no. 2, pp. 176-185, 2020.

19. [D. Khvostenko et al., "Cyclic mechanical loading promotes bacterial penetration along composite restoration marginal gaps.," Dent. Mater., vol. 31, no. 6, pp. 702-710, Jun. 2015.

20. A. D. Loguercio, M. Rezende, M. F. Gutierrez, T. F. Costa, A. Armas-Vega, and A. Reis, "Randomized 36-month follow-up of posterior bulk-filled resin composite restorations.,” J. Dent., vol. 85, pp. 93-102, Jun. 2019..

21. N. Krämer, C. Reinelt, G. Richter, A. Petschelt, and R. Frankenberger, "Nanohybrid vs. fine hybrid composite in Class II cavities: clinical results and margin analysis after four years.,” Dent. Mater., vol. 25, no. 6, pp. 750-759, Jun. 2009.

22. L. Gregor, L. Dorien, T. Bortolotto, A. J. Feilzer, and I. Krejci, "Marginal integrity of low-shrinking versus methacrylate-based composite: effect of different one-step self-etch adhesives.," Odontology, vol. 105, no. 3, pp. 291-299, Jul. 2017.

23. A. Peutzfeldt, S. Mühlebach, A. Lussi, and S. Flury, "Marginal Gap Formation in Approximal 'Bulk-Fill' Resin Composite Restorations After Artificial Ageing.," Oper. Dent., vol. 43, no. 2, pp. 180-189, 2018

24. X. Zhou et al., "Development and status of resin composite as dental restorative materials," J. Appl. Polym. Sci., vol. 136, no. 44, p. 48180, 2019.

25. M. M. de A. C. VELO, L. V. B. F. COELHO, R. T. BASTING, F. L. B. do AMARAL, and F. M. G. FRANÇA, "Longevity of restorations in direct composite resin: Literature review," RGO-Revista Gaúcha Odontol., vol. 64, no. 3, pp. 320-326, 2016.

26. A. Hervás-García, M. A. Martínez-Lozano, J. Cabanes-Vila, A. Barjau-Escribano, and P. Fos-Galve, "Composite resins. A review of the materials and clinical indications.," Med. Oral Patol. Oral Cir. Bucal, vol. 11, no. 2, pp. E215-20, Mar. 2006.

27. G. C. Lopes, L. C. C. Vieira, and E. Araujo, "Direct composite resin restorations: a review of some clinical procedures to achieve predictable results in posterior teeth.," J. Esthet. Restor. Dent. Off. Publ. Am. Acad. Esthet. Dent. ... [et al.], vol. 16, no. 1, pp. 19-31; discussion 32, 2004.

28. N. Malhotra, M. Kundabala, and A. Shashirashmi, "Strategies to overcome polymerization shrinkage--materials and techniques. A review,” Dent. Update, vol. 37, no. 2, pp. 115-118,120-122,124-125, 2010.
29. R. M, G. S. Sajjan, K. B N, and N. Mittal, "Effect of different placement techniques on marginal microleakage of deep class-II cavities restored with two composite resin formulations.," J. Conserv. Dent., vol. 13, no. 1, pp. 9-15, Jan. 2010.

30. S. P. Mantri and S. S. Mantri, "Management of shrinkage stresses in direct restorative light-cured composites: a review.," J. Esthet. Restor. Dent. Off. Publ. Am. Acad. Esthet. Dent. ... [et al.], vol. 25, no. 5, pp. 305-313, Oct. 2013.

31. V. Chandrasekhar, L. Rudrapati, V. Badami, and M. Tummala, "Incremental techniques in direct composite restoration.,” J. Conserv. Dent., vol. 20, no. 6, pp. 386-391, 2017.

32. Y. Kwon, J. Ferracane, and I.-B. Lee, "Effect of layering methods, composite type, and flowable liner on the polymerization shrinkage stress of light cured composites.," Dent. Mater., vol. 28, no. 7, pp. 801-809, Jul. 2012.

33. D. Garcia, P. Yaman, J. Dennison, and G. Neiva, "Polymerization shrinkage and depth of cure of Bulk-Fill flowable composite resins.," Oper. Dent., vol. 39, no. 4, pp. 441-448, 2014.

34. L. C. Cidreira Boaro et al., "Clinical performance and chemical-physical properties of Bulk-Fill composites resin - a systematic review and meta-analysis," Dent. Mater., vol. 35, no. 10, pp. e249-e264, 2019.

35. F. I. Alkhudhairy, "The effect of curing intensity on mechanical properties of different bulk-fill composite resins.," Clin. Cosmet. Investig. Dent., vol. 9, pp. 1-6, 2017.

36. V. H. Grandi et al., "Microtensile Bond Strength and Microhardness of Composite Resin Restorations Using a Sonic-Resin Placement System.," Braz. Dent. J., vol. 28, no. 5, pp. 618-623, 2017.

37. A. Alrahlah, N. Silikas, and D. C. Watts, "Post-cure depth of cure of Bulk-Fill dental resin-composites.," Dent. Mater., vol. 30, no. 2, pp. 149-154, Feb. 2014.

38. M. C. G. Erhardt, M. Goulart, R. C. Jacques, J. A. Rodrigues, and C. S. Pfeifer, "Effect of different composite modulation protocols on the conversion and polymerization stress profile of bulk-filled resin restorations.," Dent. Mater., vol. 36, no. 7, pp. 829-837, Jul. 2020.

39. M. Daronch, F. A. Rueggeberg, M. F. De Goes, and R. Giudici, "Polymerization kinetics of pre-heated composite.," J. Dent. Res., vol. 85, no. 1, pp. 38-43, Jan. 2006.

40. K. Nada and O. El-Mowafy, "Effect of precuring warming on mechanical properties of restorative composites., Int. J. Dent., vol. 2011, p. 536212, 2011 
41. M. Daronch, F. A. Rueggeberg, and M. F. De Goes, "Monomer conversion of pre-heated composite.," J. Dent. Res., vol. 84, no. 7, pp. 663-667, Jul. 2005.

42. M. E. Ebrahimi Chaharom et al., "Effect of preheating on the cytotoxicity of bulk-fill composite resins., J. Dent. Res. Dent. Clin. Dent. Prospects, vol. 14, no. 1, pp. 19-25, 2020.

43. Z. Metalwala et al., "Rheological properties of contemporary nanohybrid dental resin composites: The influence of preheating," Polym. Test., vol. 72, pp. 157-163, 2018.

44. A. A. Abdulmajeed, T. E. Donovan, R. Cook, and T. A. Sulaiman, "Effect of Preheating and Fatiguing on Mechanical Properties of Bulk-fill and Conventional Composite Resin.," Oper. Dent., vol. 45, no. 4, pp. 387-395, Jul. 2020.

45. A. N. A. Habib and G. H. Waly, "The degree of conversion and class II cavity microleakage of different Bulk-Fill composites placed with different restorative techniques," Futur. Dent. J., vol. 4, no. 2, pp. 231-238, 2018.

46. N. Mary, A. Subbiya, P. Vivekanandan, V. Prakash, M. Dhakshinamoorthy, and S. M. (alias) Malathi Suresh, "Effect of Composite Preheating on the Degree of Monomer Conversion, Polymerization Shrinkage and Micro Hardness of A Silorane based Composite-An In Vitro Study," Indian J. Public Heal. Res. Dev., vol. 10, p. 1615, Jan. 2019.

47. M. Patel, R. Bhatt, D. Makwani, L. Dave, and V. Raj, "Comparative evaluation of marginal seal integrity of three bulk-fill composite materials in Class II cavities: An In vitro study," Adv. Hum. Biol., vol. 8, no. 3, pp. 201-205, Sep. 2018.

48. C. Goracci et al., "Polymerization efficiency and flexural strength of low-stress restorative composites.," Dent. Mater., vol. 30, no. 6, pp. 688-694, Jun. 2014.

49. S. C. Bayne and G. Schmalz, "Reprinting the classic article on USPHS evaluation methods for measuring the clinical research performance of restorative materials," Clin Oral Investig., vol. 9, no. 4, pp. 209-214, 2005.

50. M. Laurent, P. Scheer, J. Dejou, and G. Laborde, "Clinical evaluation of the marginal fit of cast crowns--validation of the silicone replica method," J. Oral Rehabil., vol. 35, no. 2, pp. 116-122, 2008.

51. M. D. Gaintantzopoulou, V. K. Gopinath, and S. Zinelis, "Evaluation of cavity wall adaptation of bulk esthetic materials to restore class II cavities in primary molars.," Clin. Oral Investig., vol. 21, no. 4, pp. 1063-1070, May 2017.
52. J. G. Leprince, W. M. Palin, J. Vanacker, J. Sabbagh, J. Devaux, and G. Leloup, "Physico-mechanical characteristics of commercially available bulk-fill composites," J Dent., vol. 42, no. 8, pp. 993-1000, 2014.

53. E. A. Abdulkadir and M. M. Nayif, "Influence of Adhesive Systems on Microleakage Pattern of Composite Resin Restorations," Al-Rafidain Dent. J., vol. 13, no. 3, pp. 547-554, Jun. 2020.

54. C. Shahidi, I. Krejci, and D. Dietschi, "In Vitro Evaluation of Marginal Adaptation of Direct Class II Composite Restorations Made of Different 'Low-Shrinkage' Systems.," Oper. Dent., vol. 42, no. 3, pp. 273-283, 2017.

55. A. R. Yazici, S. A. Antonson, Z. B. Kutuk, and E. Ergin, "Thirty-Six-Month Clinical Comparison of Bulk-Fill and Nanofill Composite Restorations.," Oper. Dent., vol. 42, no. 5, pp. 478-485, 2017.

56. J. W. V van Dijken and U. Pallesen, "Posterior bulk-filled resin composite restorations: A 5-year randomized controlled clinical study.," J. Dent., vol. 51, pp. 29-35, Aug. 2016.

57. Y. Bayraktar, E. Ercan, M. M. Hamidi, and H. Çolak, "Oneyear clinical evaluation of different types of bulk-fill composites.,” J. Investig. Clin. Dent., vol. 8, no. 2, May 2017.

58. V. S. S. Gamarra, G. A. Borges, L. H. B. Júnior, and A. M. Spohr, "Marginal adaptation and microleakage of a bulk-fill composite resin photopolymerized with different techniques.," Odontology, vol. 106, no. 1, pp. 56-63, Jan. 2018.

59. R. Haak, T. Näke, K.-J. Park, D. Ziebolz, F. Krause, and H. Schneider, "Internal and marginal adaptation of highviscosity bulk-fill composites in class II cavities placed with different adhesive strategies.," Odontology, vol. 107, no. 3, pp. 374-382, Jul. 2019.

60. W. C. Wagner, M. N. Aksu, A.-M. L. Neme, J. B. Linger, F. E. Pink, and S. Walker, "Effect of pre-heating resin composite on restoration microleakage.," Oper. Dent., vol. 33, no. 1,pp. 72-78, 2008.

61. J. da Costa, R. McPharlin, T. Hilton, and J. Ferracane, "Effect of heat on the flow of commercial composites.," Am. J. Dent., vol. 22, no. 2, pp. 92-96, Apr. 2009.

62. N. Choudhary, S. Kamat, T. Mangala, and M. Thomas, "Effect of pre-heating composite resin on gap formation at three different temperatures.," J. Conserv. Dent., vol. 14, no. 2, pp. 191-195, Apr. 2011. 
63. N. R. Fróes-Salgado, L. M. Silva, Y. Kawano, C. Francci, A. Reis, and A. D. Loguercio, "Composite pre-heating: effects on marginal adaptation, degree of conversion and mechanical properties., Dent. Mater., vol. 26, no. 9, pp. 908-914, Sep. 2010.

64. D. Liliany and V. Violetta, "Effect of Soft Drink on Surface Roughness of Preheated and Non-Preheated Nanohybrid Composite Resins," J. Indones. Dent. Assoc. Vol 3 No 2 Oct., 2020.

65. N. Bahbishi, W. Mzain, B. Badeeb, and H. M. Nassar, "Color Stability and Micro-Hardness of Bulk-Fill Composite Materials after Exposure to Common Beverages.," Mater. (Basel, Switzerland), vol. 13, no. 3, Feb. 2020.

66. T. T. Akalın, F. O. Bozkurt, M. Kusdemir, A. Özsoy, and M. Özcan, "Clinical Evaluation of Sonic-Activated High Viscosity Bulk-Fill Nanohybrid Resin Composite Restorations in Class II Cavities: A Prospective Clinical Study up to 2 Years," The European journal of prosthodontics and restorative dentistry, vol. 26, no. 3. Istanbul Medipol University, School of Dentistry, Department of Restorative Dentistry, Ataturk Bulvarı 27, 34083, Fatih, Unkapanı, Istanbul, Turkey., pp. 152-160, 2018.

67. M. Talukder, M. Hossain, and M. Moral, "Clinical evaluation of bulk-fill composite resin and layered composite resin restoration in class I cavity of permanent molar teeth," Bangabandhu Sheikh Mujib Med. Univ. J., vol. 11, no. 1 SE-Original Articles, Mar. 2018.

68. Y. K. Alkhadim, M. J. Hulbah, and H. M. Nassar, "Color Shift, Color Stability, and Post-Polishing Surface Roughness of Esthetic Resin Composites.," Mater. (Basel, Switzerland), vol. 13, no. 6, Mar. 2020.

69. C. P. Lepri and R. G. Palma-Dibb, "Surface roughness and color change of a composite: influence of beverages and brushing.," Dent. Mater. J., vol. 31, no. 4, pp. 689-696, 2012.

70. N. Gönülol and F. Yılmaz, "The effects of finishing and polishing techniques on surface roughness and color stability of nanocomposites,” J. Dent., vol. 40, pp. e64-e70, 2012.
71. D. Sarac, Y. S. Sarac, S. Kulunk, C. Ural, and T. Kulunk, "The effect of polishing techniques on the surface roughness and color change of composite resins.," J. Prosthet. Dent., vol. 96, no. 1, pp. 33-40, Jul. 2006.

72. H. Kocaagaoglu, T. Aslan, A. G\&\#252;rbulak, H. Albayrak, Z. Ta\&\#351;demir, and H. Gumus, "Efficacy of polishing kits on the surface roughness and color stability of different composite resins," Niger. J. Clin. Pract., vol. 20, no. 5, pp. 557-565, May 2017.

73. E.-H. Kim, K.-H. Jung, S.-A. Son, B. Hur, Y.-H. Kwon, and J.-K. Park, "Effect of resin thickness on the microhardness and optical properties of bulk-fill resin composites.," Restor. Dent. Endod., vol. 40, no. 2, pp. 128-135, May 2015.

74. D. Dionysopoulos, K. Tolidis, and P. Gerasimou, "The Effect of Composition, Temperature and Post-Irradiation Curing of Bulk-Fill Resin Composites on Polymerization Efficiency ," Materials Research , vol. 19. scielo , pp. 466-473, 2016.

75. A. A. Elkaffas, R. I. Eltoukhy, S. A. Elnegoly, and S. H. Mahmoud, "The effect of preheating resin composites on surface hardness: a systematic review and meta-analysis.," Restor. Dent. Endod., vol. 44, no. 4, p. e41, Nov. 2019.

76. K. Heck, J. Manhart, R. Hickel, and C. Diegritz, "Clinical evaluation of the Bulk-Fill composite QuiXfil in molar class I and II cavities: 10-year results of a RCT.," Dent. Mater., vol. 34, no. 6, pp. e138-e147, Jun. 2018.

77. A. Correia, A. Jurema, M. R. Andrade, A. Borges, E. Bresciani, and T. Caneppele, "Clinical Evaluation of Noncarious Cervical Lesions of Different Extensions Restored With Bulk-fill or Conventional Resin Composite: Preliminary Results of a Randomized Clinical Trial,” Oper. Dent., vol. 45, no. 1, pp. E11-E20, 2020.

78. M. Sadeghi, C. D. Lynch, and N. Shahamat, "Eighteenmonth clinical evaluation of microhybrid, packable and nanofilled resin composites in Class I restorations," J. Oral Rehabil., vol. 37, no. 7, pp. 532-537, 2010. 